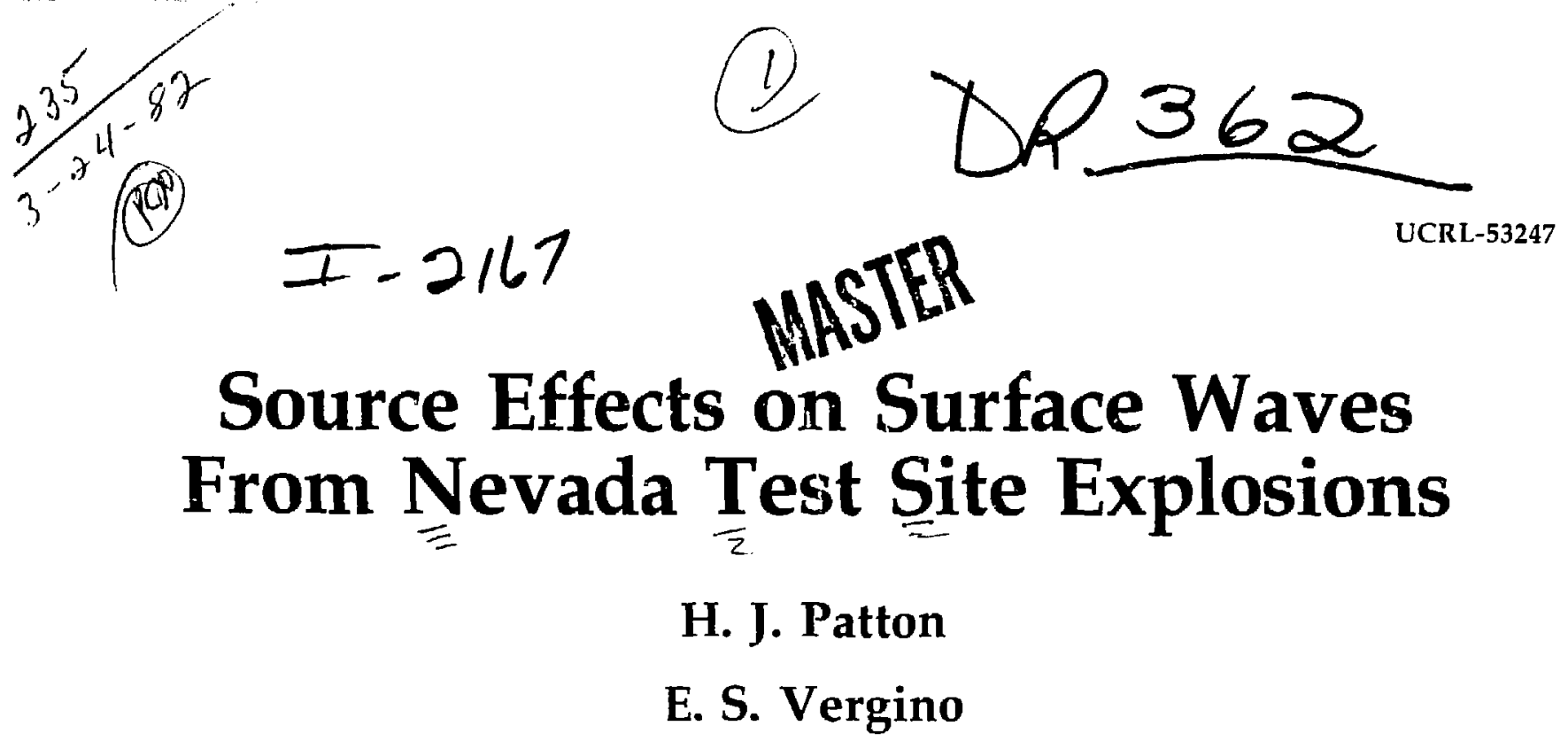

November 1981

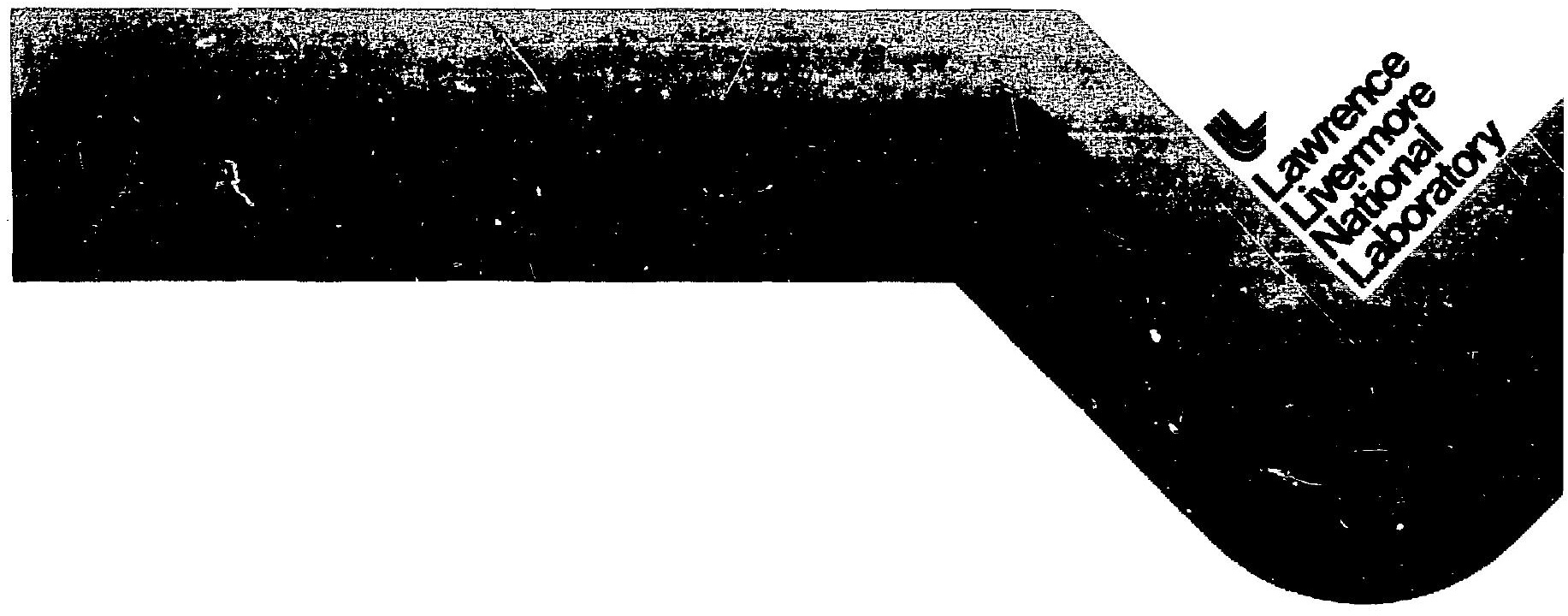


UCRL-53247

Distribution Category UC-11

UCRL- -53247

DES 2009357

\title{
Source Effects on Surface Waves From Nevada Test Site Explosions
}

\author{
H. J. Patton \\ E. S. Vergino
}

Manuscript Date: November 1981

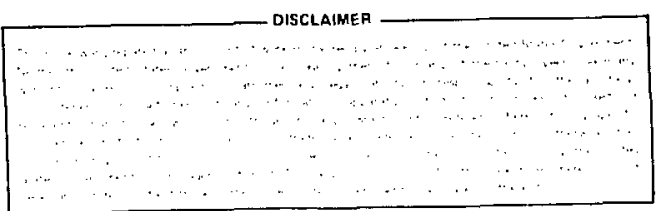

LAWRENCE LIVERMORE LABORATORY

University of California $\bullet$ Livermore, California 94550 
Abstract

Int roduction Waveform Data

Filtering of Waveform Data

Low-Pass Filtering

Narrow-Band Filtering

Spectral Analysis

Signal and Noise Spectra

Surface-Wave Source Spectra.

Source Amplitude Spectra

Source Phase Spectra

Results.

Modeling the Explosion Source

ReJuced Displacement Potential

Parameter Study

Modeling Results.

Effect of Crustal Structure

Discussion.

Secondary Sources of Seismic Waves

Spall Closure

Thrust Faulting

Tectonic Straiı Release

Discussion of yodeling Results Conclusions

Future Work

Augmented Datasets

RDP at Higher Frequencies .

Love-wave Generation . . - . • . . . . . . . . . . 53

Improved Inversion Techniques

Acknowledgments

Appendix A--Procedures for Analysis Techniques

References 
ABSTRACT

Surface waves recorded on the Lawrence Livermore National Laboratory (LLNL) digital network have been used to study five underground nuclear explosions detonated in Yucca Valley at the Nevada Test Site. The purpose of this study is to characterize the reduced displacement potential (RDP) at low frequencies and to test secondary source models of underground explosions. The observations consist of Rayleigh- and Love-wave amplitude and phase spectra in the frequency range 0.03 to $0.16 \mathrm{~Hz}$. We have found that Rayleighwave spectral amplitudes are modeled well by a RDP with little or no overshoot for explosions detonated in alluvium and tuff. On the basis of comparisons between observed and predicted source phase, the spall closure source proposed by Viecelli does not appear to be a significant source of Rayleigh waves that reach the far Eield. We tested two other secondary source models, the strike-slip, tectonic strain release model proposed by Toksöz and Kehrer and the dip-slip thrust nodel of Massé. The surface-wave observations do not provide sufficient information to discriminate between these models at the low F-values ( 0.2 to 0.8 ) obtained for these explosions. In the case of the strike-slip model, the principal stress axes inferred from the fault slip angle and strike angle are in good agreement with the regional tectonic stress field for all but one explosion, Nessel. The results of the Nessel explosion suggest a mechanism other than tectonic strain release.

\section{INTRODUCTION}

The explosion source has been the object of intensive study in seismology for aver two decades. The goals of the study are to improve the ability to discriminate between earthquakes and explosions and to estimate yield. In large part, improvements depend upon advances in the knowledge of the source physics and upon development of models of the seismic source. A good deal of the uncertainty in our present capabilities is because there is no widely accepted source model. For example, investigators are still raising questions 
about the source time function of explosions and the role of secondary sources (see Müller, ${ }^{1}$ Springer, ${ }^{2}$ and Masse ${ }^{3}$ ).

We have been studying surface waves from underground explosions detonated in Yucca Valley at the Nevada Test Site (NTS). Our purpose is to gain a better understanding of the generation of low-frequency seismic waves from explosions. This paper is a progress report of research in the past two years. We present the data in the section entitled Waveform Data. The results of filtering the data that characterize the frequency content and arrival times of low-frequency waves is given in the section entitled Filtering of Waveform Data. We carry out spectral analysis of the seismograms and calculations of surface-wave source spectra in the sections entitled Spectral Analysis and Surface-Wave Source Spectra, respectively. Our efforts to deduce the shape of the reduced displacement potential (RDP) at low Erequencies and to model three secondary sources (spall slapdown, tectonic strain release and thrust faulting) are described in the sections entitled Modeling the Explosion Source and Secondary Sources of Seismic Waves. Conclusions to date are given in the last section.

\section{WAVEFORM DATA}

We considered for study the seismograms from 11 underground nuclear ëpiosions detonated at NTS. Table 1 contains pertinent geologic and seismic data about these explosions. The surface waves from only 5 of the 11 explosions could be analyzed because of the high microseismic noise level in the winter and spring months, as is shown in the section on spectral Analysis. We indicate in Table 1 the explosions that were precluded from study using long-period surface waves. Of the five studied in this report, two explosions (Hearts and Pyramid) were below the water table and the rest were above. All the explosions occurred in Yucca Valley (see Fig. 1).

The waveforms were digitally recorded on the four Lawrence Livermore National Laboratory (LLNL) seismic stations surrounding NCS. As may be seen in Fig. 2, these stations are between 200 and $400 \mathrm{~km}$ from NTS and are approximately $90^{\circ}$ apart in azimuth. The three-component seismograph system at each station is flat to velocity between $30 \mathrm{~s}$ and $10 \mathrm{~Hz}$ (see Denny ${ }^{4}$ ).

The broadband waveforms for the Nessel. explosion are shown in Figs. 3 to 6 , where the time frame is set so that $0.0 \mathrm{~s}$ is the origin time of the explosior. Positive deflection on the vertical component record indicates 
Cable 1. Epicentral and pertinent geologic data for explosions in this study.

\begin{tabular}{|c|c|c|c|c|c|c|c|c|}
\hline Date & $\begin{array}{c}\text { Name of } \\
\text { explosion }\end{array}$ & $\begin{array}{l}\text { Latitude } \\
\left({ }^{\circ} \mathrm{N}\right)\end{array}$ & $\begin{array}{l}\text { Long itude } \\
\qquad\left({ }^{\circ} \mathrm{W}\right)\end{array}$ & Medium ${ }^{a}$ & $\begin{array}{c}\text { Depth of } \\
\text { burial } \\
\text { (m) }\end{array}$ & $\begin{array}{l}0 P z \\
(\text { iII })\end{array}$ & $\begin{array}{l}\mathrm{D}_{\mathrm{H}} 2 \mathrm{O} \\
(\mathrm{m})\end{array}$ & $\begin{array}{r}b \\
m_{b}\end{array}$ \\
\hline $08 / 03 / 79$ & Burzet & 37.08 & 116.07 & $A L$ & 450 & 849 & 510 & 4.5 \\
\hline $03 / 08 / 79$ & Offshore & 37.02 & 116.01 & ru & 396 & 660 & 467 & 4.8 \\
\hline $08 / 29 / 79$ & Nessel & 37.12 & 116.07 & $A L$ & $46 \div$ & 975 & 341 & 4.7 \\
\hline $09 / 06 / 79$ & Hearts & 37.09 & 116.05 & $\mathrm{TU}$ & 640 & 1071 & 507 & 5.8 \\
\hline $09 / 08 / 79$ & Pera ${ }^{C}$ & 37.15 & 116.04 & Tu & 200 & 503 & 553 & $3.7^{\mathrm{d}}$ \\
\hline $11 / 29 / 79$ & Backgammon ${ }^{C}$ & 36.99 & 116.02 & $A L$ & 229 & 1120 & 471 & 3.8 \\
\hline $12 / 14 / 79$ & $A z u L^{C}$ & 37.14 & 116.06 & $\mathrm{AL}$ & 205 & 755 & 561 & $3.6^{13}$ \\
\hline $0 ? / 28 / 80$ & Tarko ${ }^{C}$ & 37.13 & 116.09 & $\mathrm{AL}$ & 369 & 670 & 564 & 4.4 \\
\hline $03 / .58 / 80$ & Norbo ${ }^{c}$ & 37.18 & 116.08 & $\Gamma U$ & 271 & 402 & 553 & 3.9 \\
\hline $94 / 03 / 80$ & Liptaur ${ }^{c}$ & 37.15 & 116.08 & $\mathrm{AL}$ & 417 & 834 & 581 & 4.7 \\
\hline $04 / 16 / 80$ & Pyramid & 37.10 & 116.03 & $\Gamma \mathrm{J}$ & 379 & 783 & 540 & 5.3 \\
\hline
\end{tabular}

a AL denotes alluvium; TU denotes tufE.

b Budy-wave magnitudes from the Earthquake Data Reports (EDR) of the U.S. Geological Survey.

$C$ Denutes events for which signal to noise ratio at Erequencies below $0.1 \mathrm{~Hz}$ precluded analysis of surface waves.

d. 
$10^{\prime}$

$$
\text { 7'30' }
$$

$5^{\prime}$

$2^{\prime} 30^{\prime \prime}$

$116^{\circ} 00^{\prime}$

$57^{\prime} 30^{\prime \prime}$

$55^{\circ}$

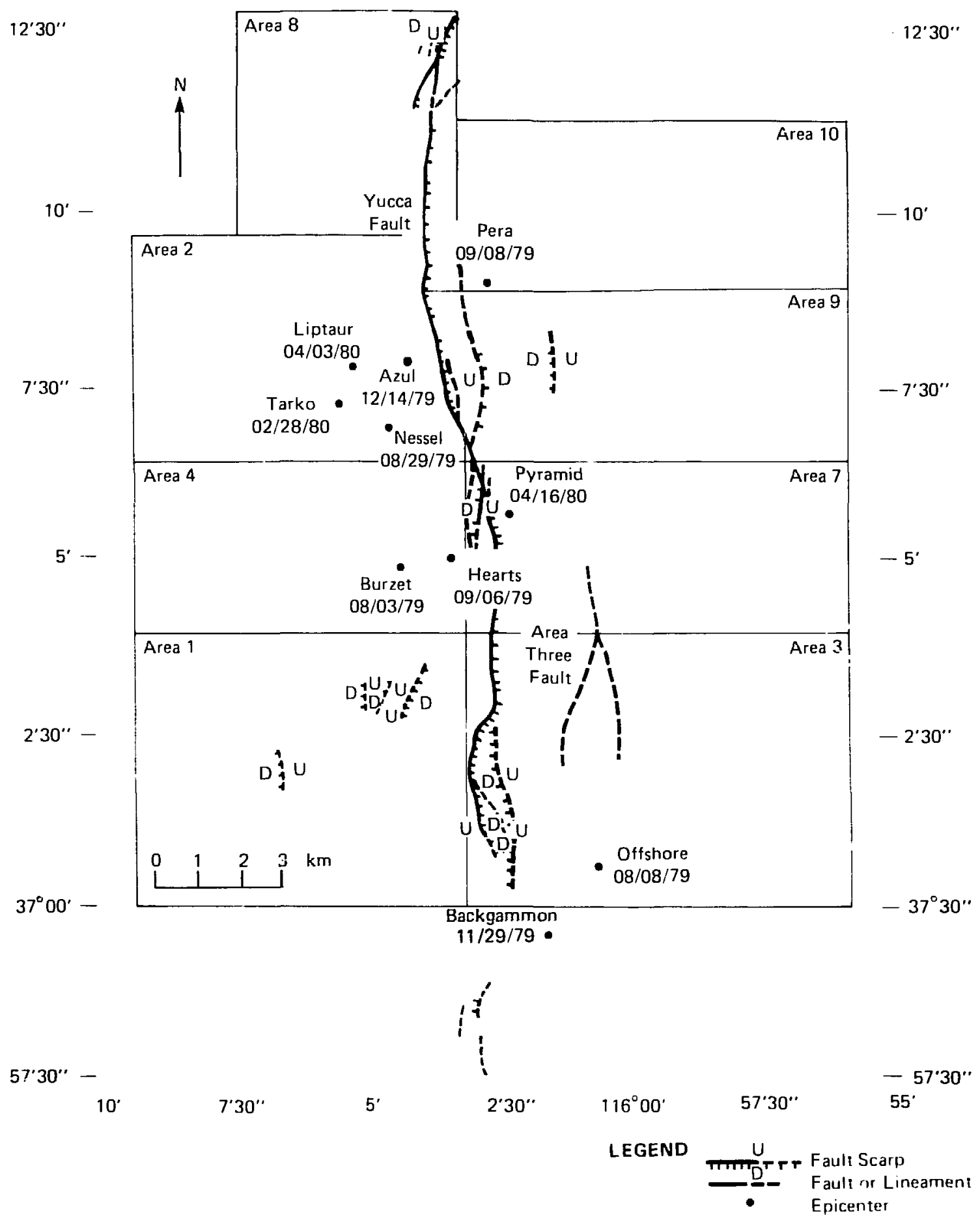

Figure 1. Map of Yucca Valley showing locations of explosions studied in this paper. Fault traces are taken Erom U.S.G.S. geologi $=7.13 .5$ 


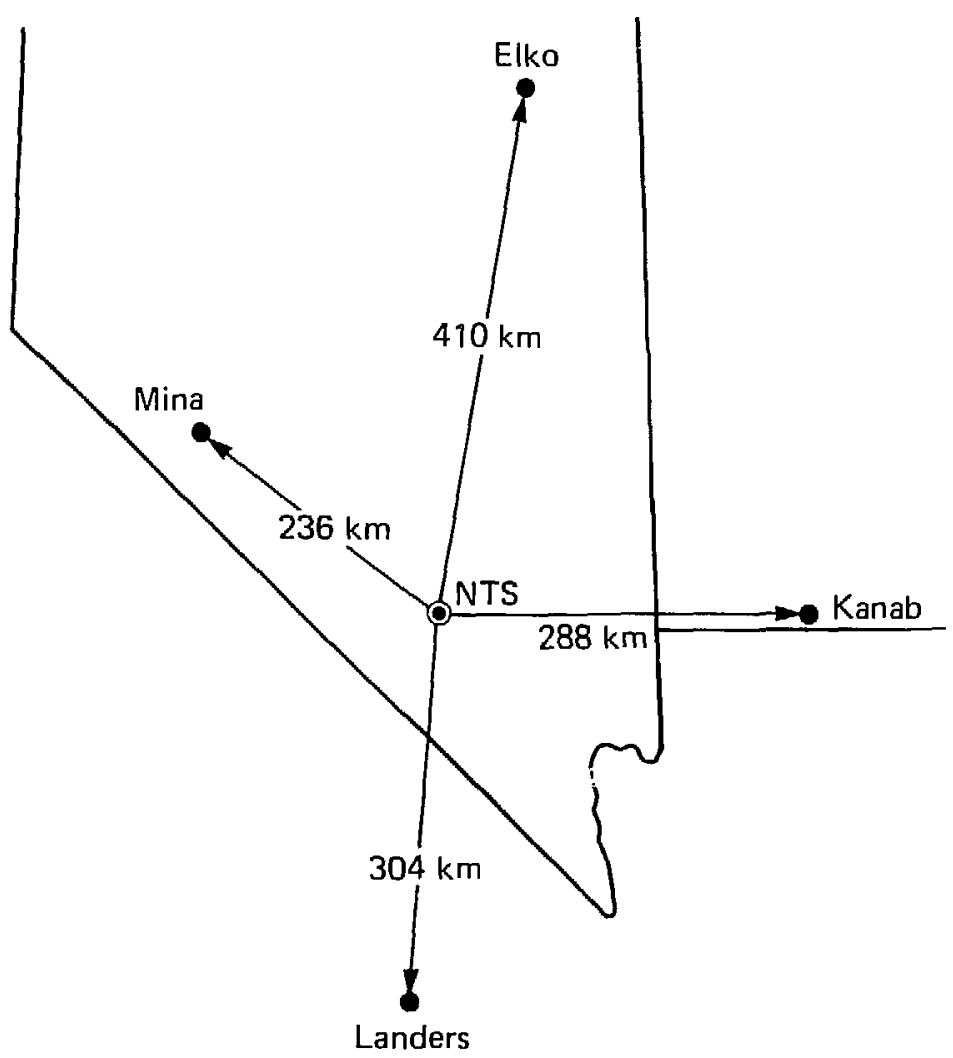

Figure 2. Locations of Lawrence Livermore iNational Laboratory broadband seismograph stations used in this study. 


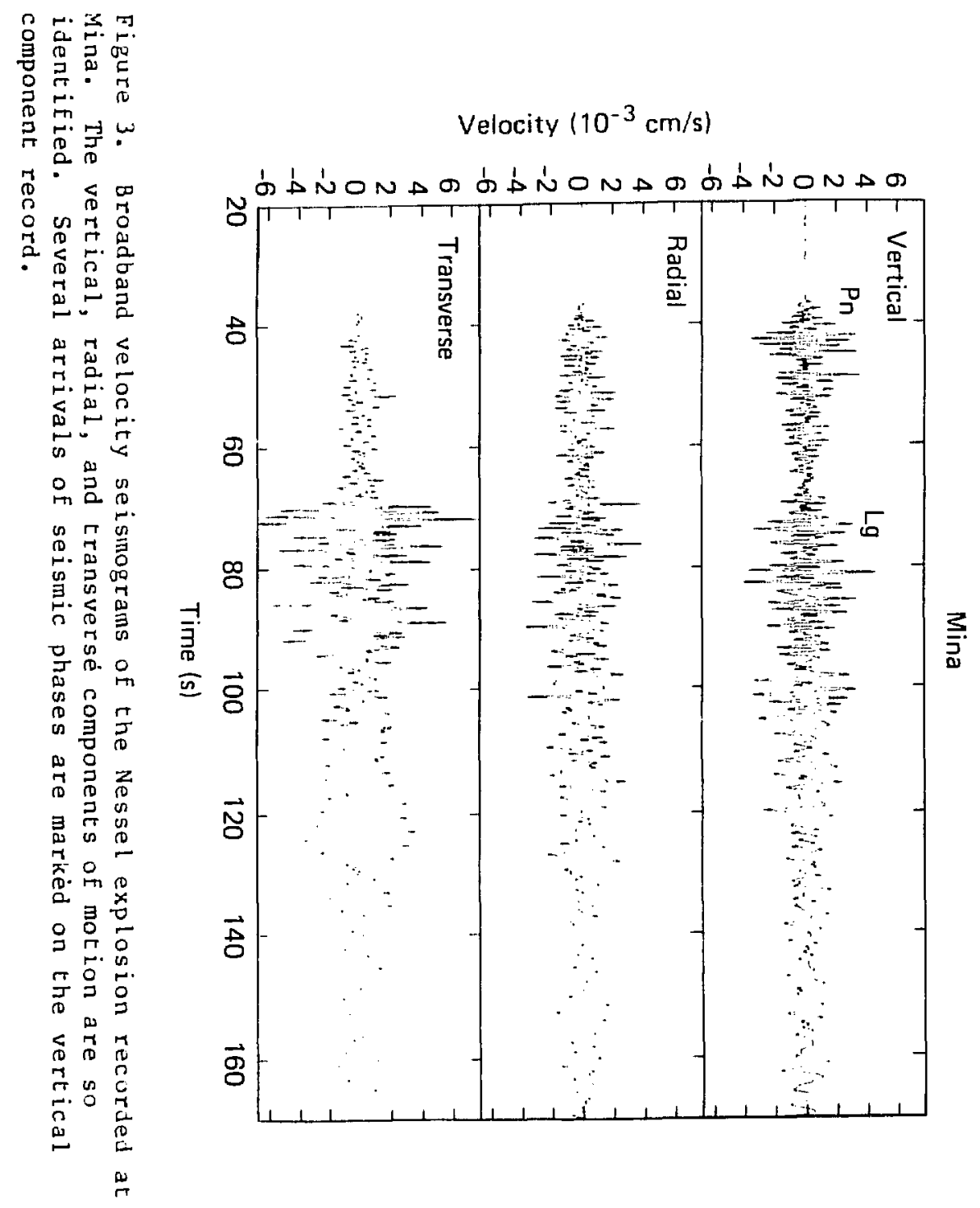




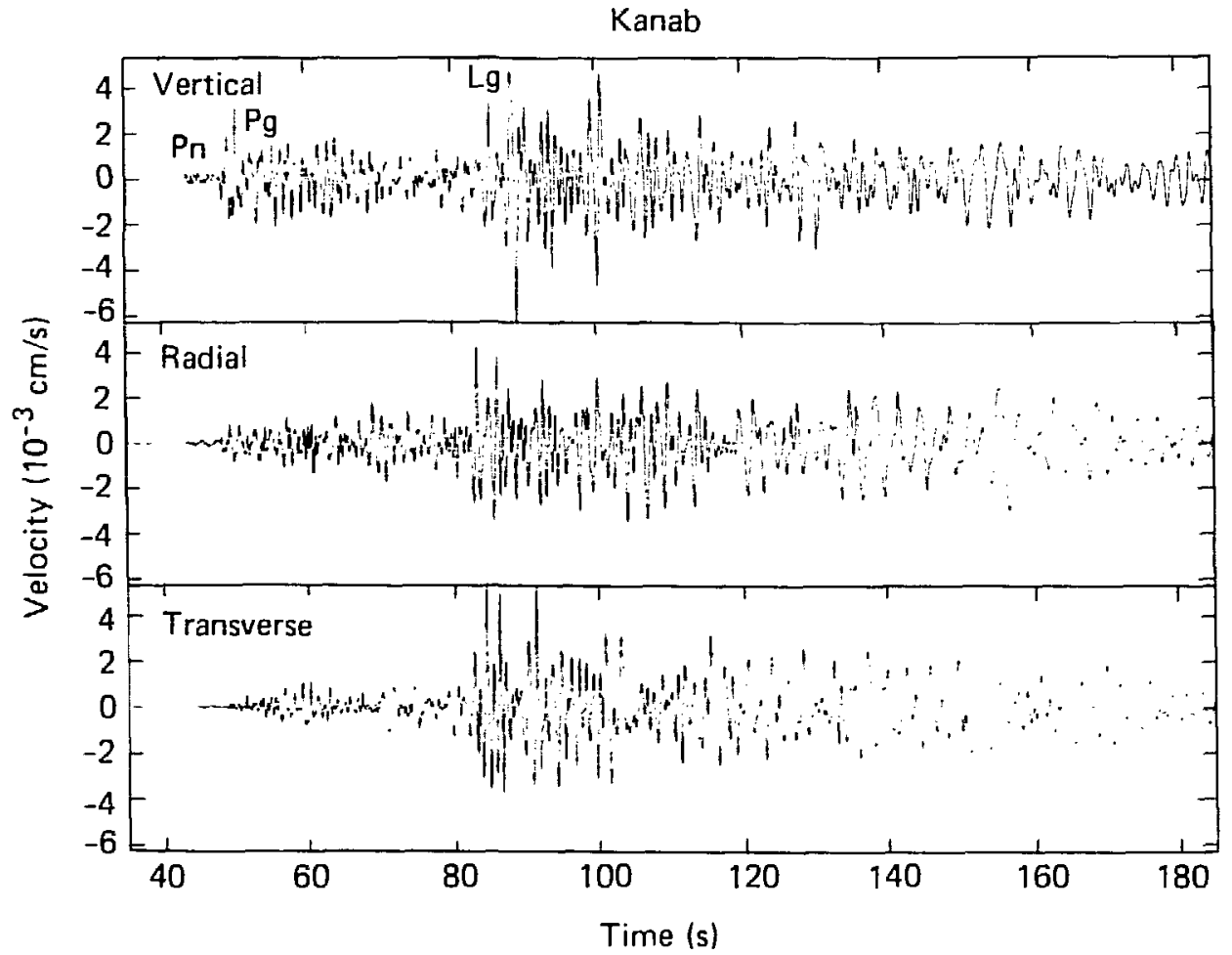

Fibure 4. Broadband velocity seismograms of the Nessel explosion recorded at Kanab. The vertical, radial, and transverse components of motion are so identified. Several arrivals of seismic phases are marked on the vertical component record. 


\section{Landers}

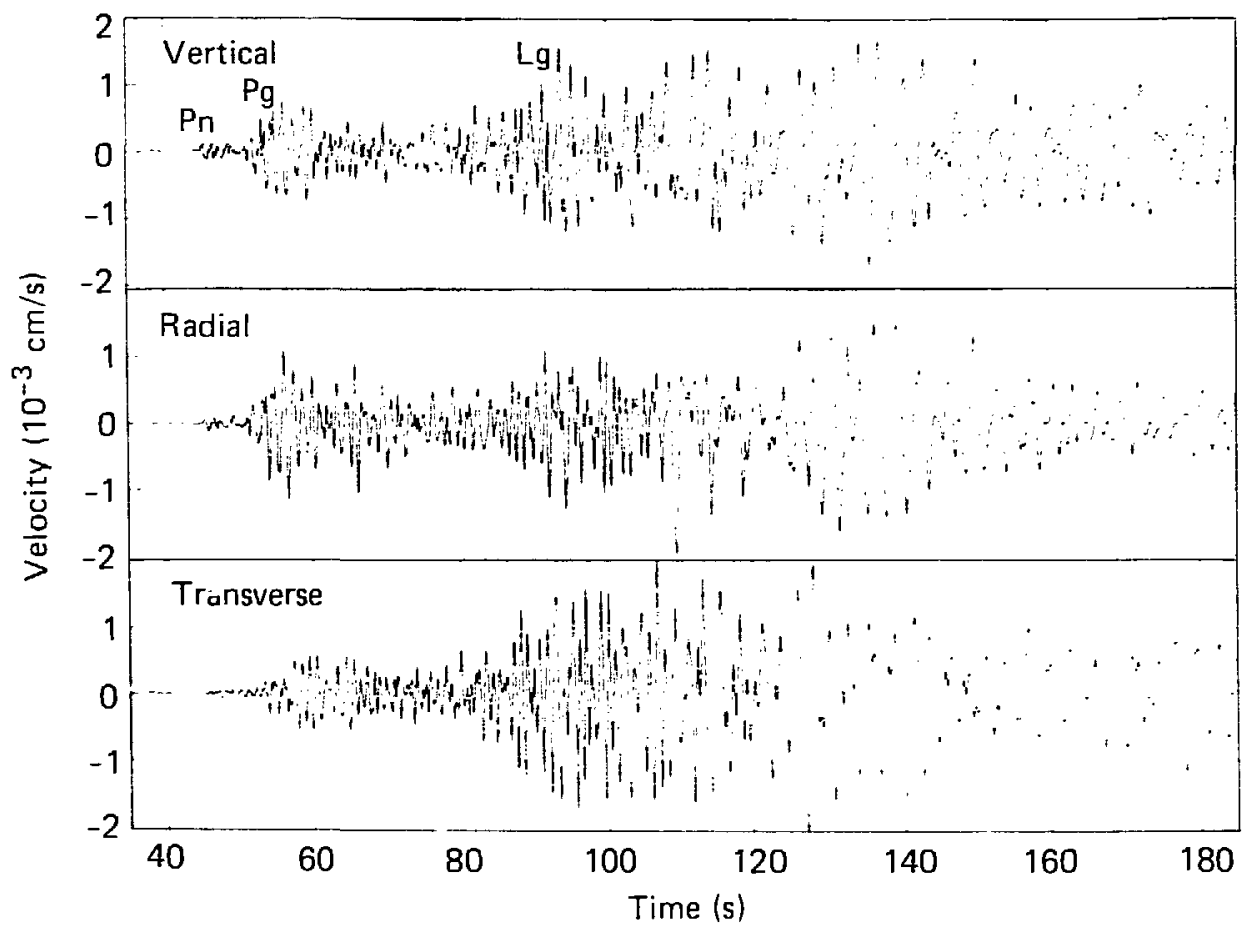

Fifure 5. Bruadband velocity seismograms. of the Nessel explosion recorded at Landers. The vertical, radial, and transverse components of motion are so identified. Several arrivals of seismic phases are marked on the vertical component record. 


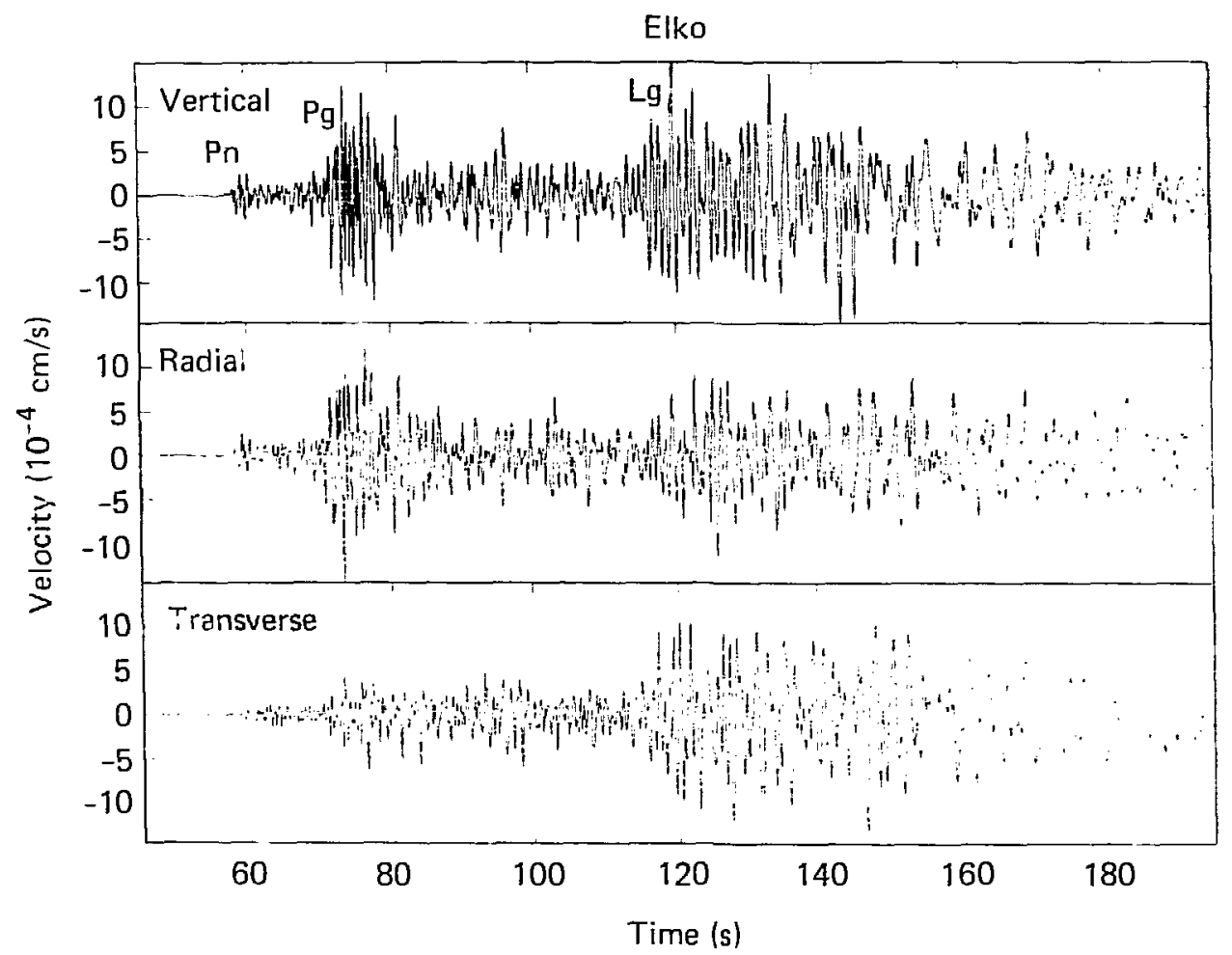

Figure 6. Broadband velocity seismograms of the Nessel explosion recorded at Elko. The vertical, radial, and transverse components of motion are so identified. Several arrivals of seismic phases are marked on the vertical $c$ aponent record. 
upward ground motion; on the radial component, positive indicates grourd notion away from NTS; and on the transverse compcnent, positive indicates counterclockwise motion.

We mark the arrival time of $P_{n}$ and the arrival times of the principal wave packets of $\mathrm{rg}_{\mathrm{g}}$ and $\mathrm{L}_{\mathrm{g}}$ on these figures. The arrivals of higin Erequency energy in $\mathrm{Pn}, \mathrm{Pg}$, and $\mathrm{Lg}$ are very clear on these broadband records. In this study, particular attention has been paid to the surface-wave arrivals. This includes both vertical and transverse Lg waves, Rayleigh waves, and Love waves. Although Lg waves travel faster than Rayleigh and Love waves, their time separations are snall for the epicentral distances in this study.

FILTERING OF WAVEFOR:! DATA

In order to charactel ze the surface waves in terms of frequency content and arrival times of significant energy, the waveforms have been filcered. We will denonstrate that $\mathrm{Lg}$ waves and surface waves can be separated on tne basis of Erequency content, although they are not separated in ime. The importance of Eraquency separation will be evident when spectra are interpreted later in this study.

LON-PASS FILTERING

The procedure for low-pass filtering is described in detail in Appendix A. The filters are computed in the Erequency domain and applied to the Fourier spectrum of the signal in a selected time window (ase Table 2). We selected filters with corner frequencies at $0.2,0.33$, and $0.5 \mathrm{~Hz}$ For vertical component waveforms and at $0.125,0.2,0.33$, and $0.5 \mathrm{~Hz}$ for transverse component waveforms. The filters have zero phase.

Examples of the low-pass filtering of signals recorded at Landers are given in Fig. 7. The Lg wave travels at about $3.4 \mathrm{~km} / \mathrm{s}$ and nost of its energy is in the frequencles higher than $0.33 \mathrm{~Hz}$. The Airy phase of the fundamentalmode Rayleigh wave travels at about $2.8 \mathrm{kin} / \mathrm{s}$ and is the predominant arrival Eor periods longer than $5 \mathrm{~s}$ (Fig. $7 \mathrm{a}$ ). This is also crue ol the Love waves which travel at about $3.1 \mathrm{~km} / \mathrm{s}$ (Fig. 73). Tnere appears to de considerable scattered energy for wave periods between 2 and $5 \mathrm{~s}$, which could be due to 
Table L. Time windows for low-pass filtering.

\begin{tabular}{lcc}
\hline Stations & $\begin{array}{c}\text { Vertical } \\
\text { component } \\
(\mathrm{s})\end{array}$ & $\begin{array}{c}\text { Transverse } \\
\text { comp sneut } \\
(\mathrm{s})\end{array}$ \\
\hline Mina & 60 to 130 & 55 to 130 \\
Kanab & 80 to 160 & 60 to 150 \\
Landers & 80 to 160 & 65 to 155 \\
Elko & 110 to 190 & 95 to 185 \\
\hline
\end{tabular}

multipathing of the fundamental-mode Rayleigh waves and scattering of niginer modes. The most important feature is that for periods longer tuan $j$ s the seismic energy is carried principally by the fundamental-mode Rayleigh and Love waves. This is confirmed from the group velocity dispersion which will be pre:iented in the next section. For periods less than $5 \mathrm{~s}$ there is appreciable energy arriving in addition to that carried by the fundamentalmode surface waves.

NARROW-BAND FILTERING

We nave narrow-band-filtered the data -o study the arrival times of surface-wave energy as a function of frequency. The results will be used to measure group velocity dispersion and to determine the frequencies where multipathing and seismic wave scattering become important.

The procedure for narrow-band filtering is described in detail in Appendix A. Again the filters are computed in the frequency domain and applied to the Fourier spectra of waveforms for the time windows in rable 3. The narrow-band filters were computed using a Gaussian function as described by Dziewonski et al. ${ }^{\circ}$ The center frequencies were selected at $0.33,0.25$, $0.22,0.20,0.18,0.107,0.125,0.10,0.033$, and $0.0625 \mathrm{~Hz}$. The filcers nave zero pnase.

Fi itered traces for the vertical and transverse components at the handers station are shown in Figs. 8 and 9, respectively. From the plots in these figures and others like them, it has been found that for center persods longer than about $j \mathrm{~s}$ the seismic energy arrives in distinct wave groups. At shorter 

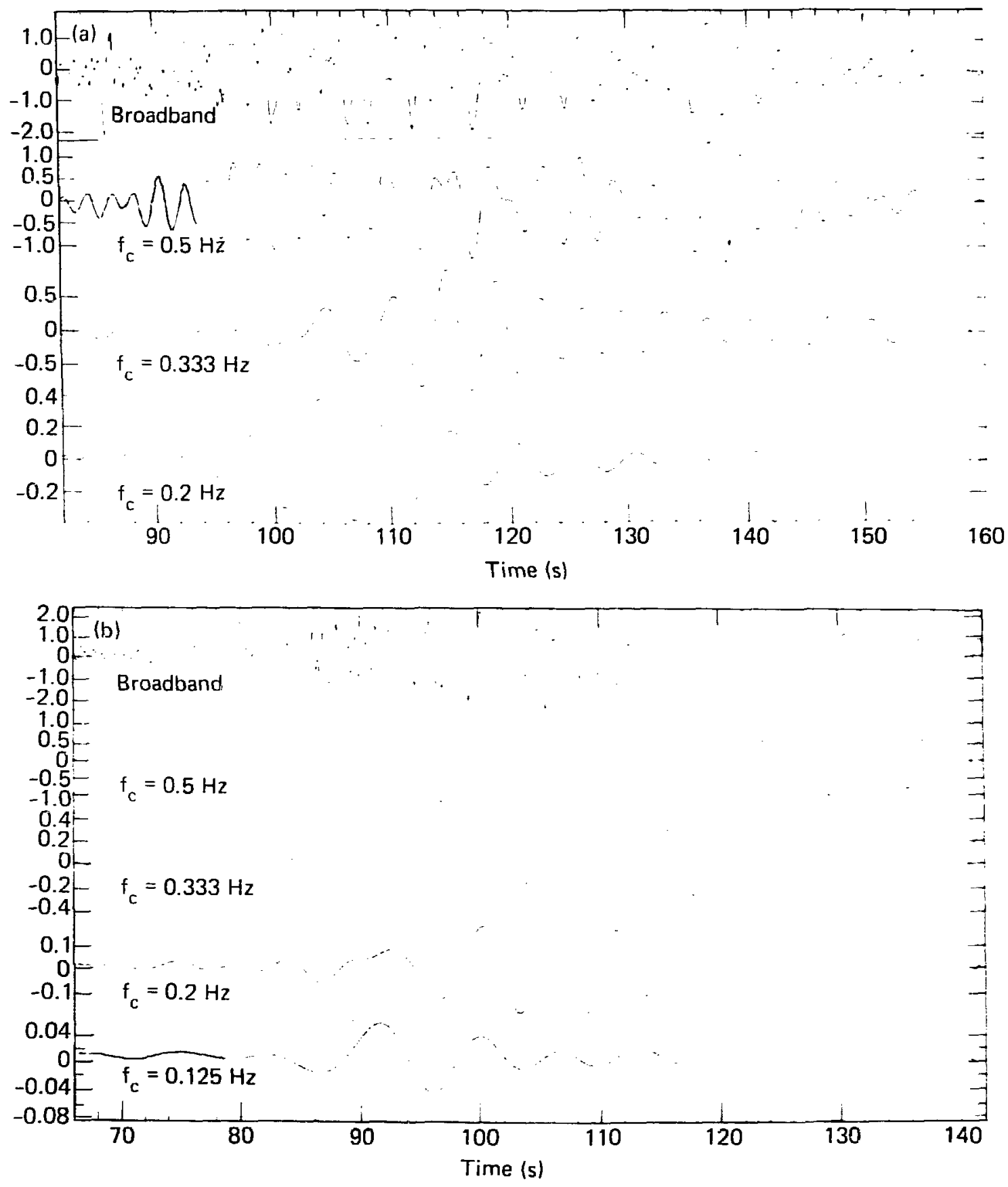

Eizite. Low-pass digital filtering of the broadband record shown in the top $i=a n e \partial f(a)$ and (b). Figure (a) is the vertical component of motion at ia $7:=5$ for the offshore explosion with the filter corner placed at 0.5 , i.j3;, and $0.2 \mathrm{~Hz}$. Figure (b) is the transverse componeat of motion with the $\therefore:$ : zorner placed at $0.5,0.333,0.2$, and $0.125 \mathrm{~Hz}$. 
Table 3. Tine windows for narrow-band

filtering and spectral analysis.

\begin{tabular}{lll} 
Stations & $\begin{array}{c}\text { Vertical } \\
\text { component } \\
(s)\end{array}$ & $\begin{array}{c}\text { Transverse } \\
\text { component } \\
(\mathrm{s})\end{array}$ \\
\hline Mina & 60 to 210 & 55 to 195 \\
Kanad & 70 to 220 & 50 to 200 \\
Landers & 70 to 220 & 50 to 200 \\
Elko & 110 to 200 & 85 to 235
\end{tabular}

periods there are multiple arrivals witn signiticant anplicude. laese ocher short-period arrivals are probably ingher-inode and mulcipatnirg surface waves. Of all stations, Landers shows the least contanination by nultiple arrivals.

The velucity of wave groups were determined oy first measuring the drrival time of the center of the wave group. This cine is adjusted ror the group delay time through the seismometer system (sie Rodgers et al. ${ }^{7}$ ). Then til.: adjusted times are divijed into the epicentral distance to outain the sroup velocity of Rayleign/Love waves for a frequency which is the center frequency of the narrow-dand filter. We note that the correction for group delay conoun the seismometer system was small for the frequencies of interest in this study. The largest delay is about $3 \mathrm{~s}$ at the lowest frequency, $0.06 \mathrm{~Hz}$. The Rayleigh-wave dispersion curves for each station path are shown in Fig. 10(a). The Love-wave dispersion curves are shown in Fig. $10(b)$. The results from just the Hearts explosion are plotted. There was quite good agreement between the results from explosions Hearts and Nessel. The Loveand Rayleigh-wave group velocities at periods longer than 8 s are significantly lower on the path to Mina. Lower Rayleigh-wave phase velocities are also observed on this path (see Patton ${ }^{8}$ ). We believe these velocitias are caused by lower velocities and/or thicker crust in the Basin and Range structure northwest of NTS.

Theoretical group velocity curves for the earth structure proposed by Priestley and Brune ${ }^{9}$ for the Basin and Range Province of the westeru U.S. 


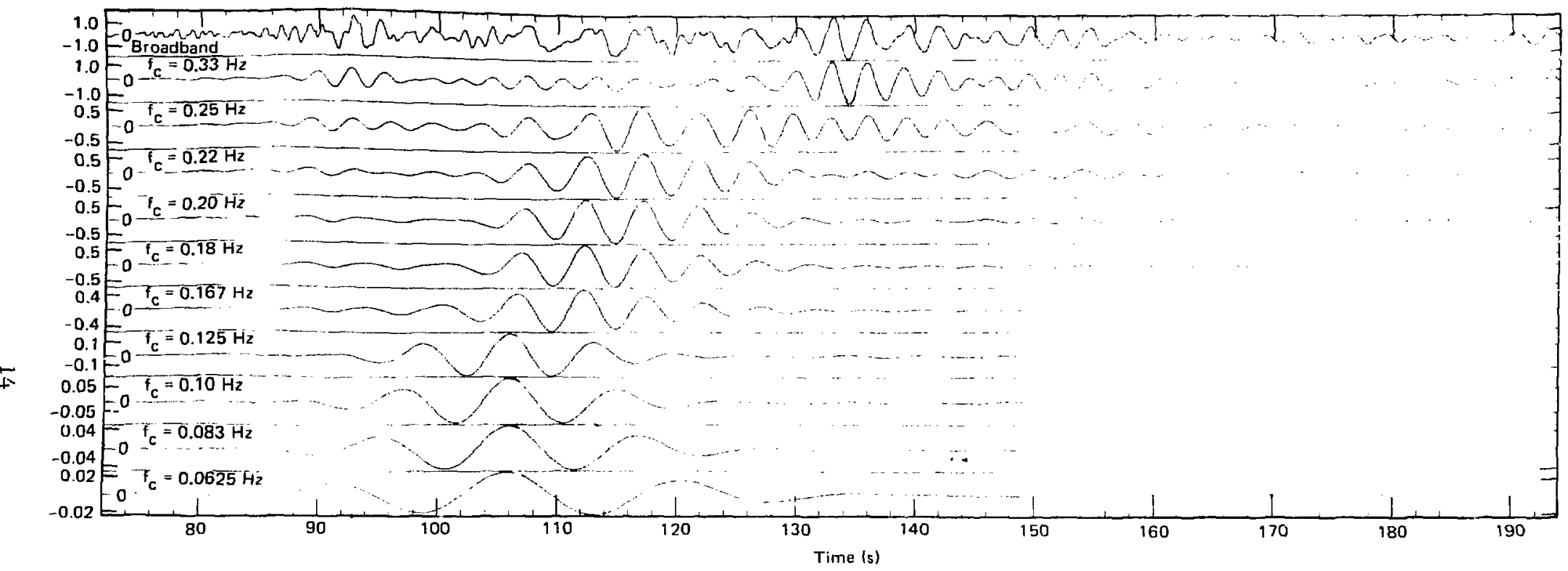

Eigure 8. Narrow-band filtering of the broadband record shown in the top frame. Beneath top frame are Eiltered records with the center Erequency set at $0.33,0.25,0.22,0.20,0.18,0.167,0.125,0.10,0.083$, and $0.0625 \mathrm{~Hz}$. Landers' vertical component recording of the Hearts explosion is shown in the top frame. 


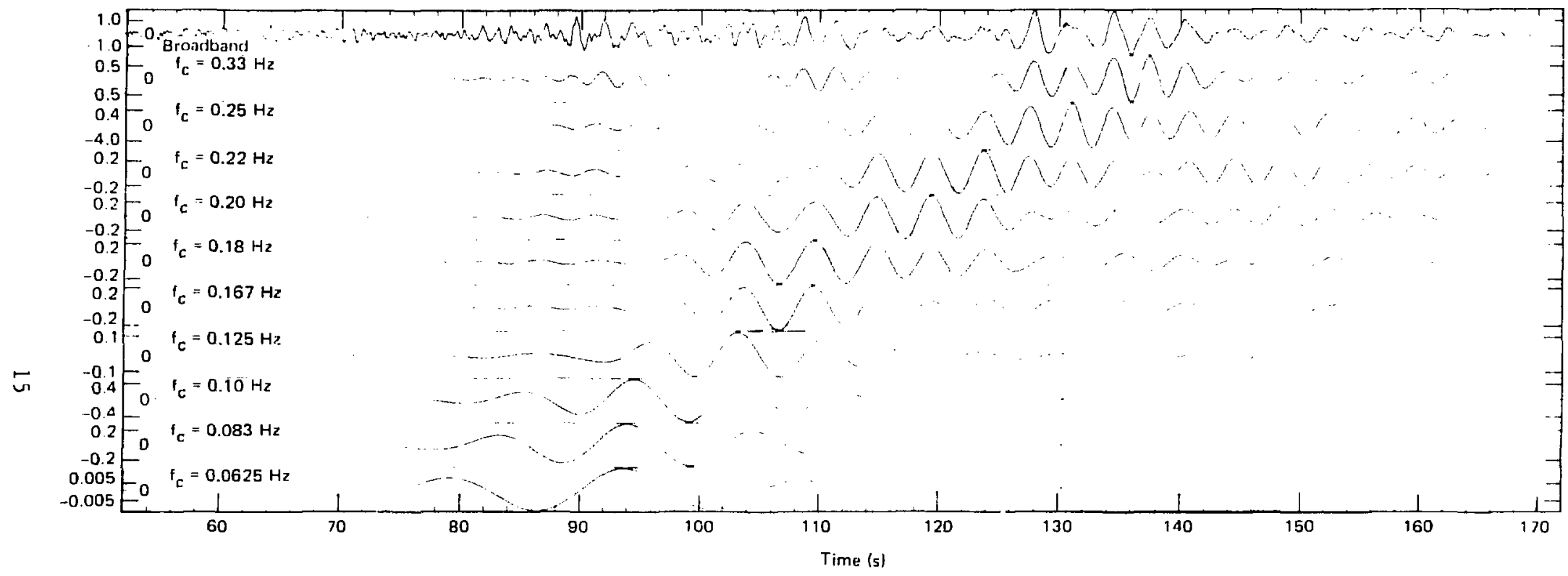

Figure 9. Narrow-band filtering of the broadband record shown in the top frame. Beneath top frame are filtered records with the center frequency set at $0.33,0.25,0.22,0.20,0.18,0.167,0.125,0.10,0.183$, and $0.0625 \mathrm{~Hz}$. Landers' transverse component recording of the Hearts explosion is shown in the top frame. 

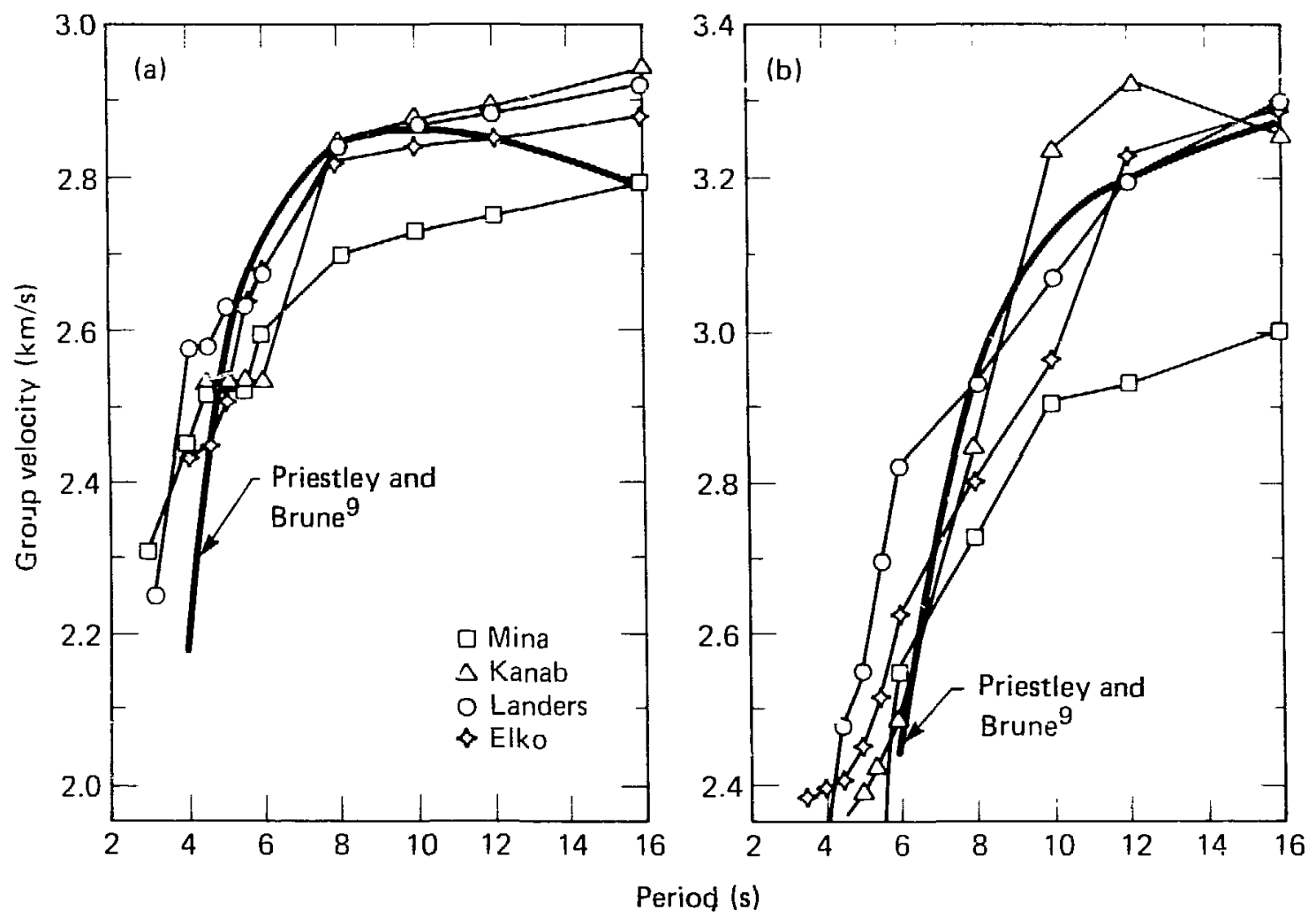

Figure 10. (a) Rayleigh-wave group velocity dispersion curves for paths between the Nevada Test Site and each of the Lawrence Livermore National Laboratory seismograph stations. (b) Love-wave group velocity dispersion curves for paths between the Nevada Test Site and eaun of the Lawreace Livermore National Laboratory seismograph stations. 
are snown with the observations in Fig. 10. The general agreement of our ubservations with the theoretical curves supports the identification of taste seismic waves as Rayleigh and Love waves for the period range 4 to $20 \mathrm{~s}$.

\section{SPECTRAL ANALYSIS}

The results of the previous section indicate that the seismic energ at Erequencies below $0.2 \mathrm{~Hz}$ is contained in the Eundamental-mode Rayleigh/Love waves generated by the explosions. This is important because the low-frequen: spertra of broadband waveforms can be interpreted as due to the motion of a single type of seismic wave, either Rayleigh or Love waves. Analysis of Eruquencies higher than $0.2 \mathrm{~Hz}$ will require multi-mode separation techniques, filtering of secondary arrivals, and/or more selective windowing of seismic energy before it is possible to make interpretations.

With the high frequency limit so determined, we turn to the low frequency limit of our analysis. This is determined by two factors: (1) the low frequency corner of the seismograph response and (2) the level of background noise relat've to signal strength. The free period of the seismometer determines the corner frequency. The free period is $30 \mathrm{~s}$ for all components and all stations. The second factor was considered by analyzing the spectra of noise samples as discussed below.

\section{SIGNAL AND NOISE SPECTRA}

The procedure for the spectral analysis of signals and noise samples is described in detail in Appendix A. Time windows for the signals are given in Table 3. Noise windows, the same length as the signal windows, were selected before the onset of p-waves. A few examples of amplitude spectra of signal and noise are given in Fig. 11. Arrows indicate the lowest frequency with signal significantly above the noise. All signal spectra were compared with the noise spectra to estimate the lowest frequency with satisfactory signal to noise ratio. It should be noted that the background noise in the winter and spring months (December through April) was an order of magnitude above the noise level during the summer months. The microseismic noise was large enough that the 


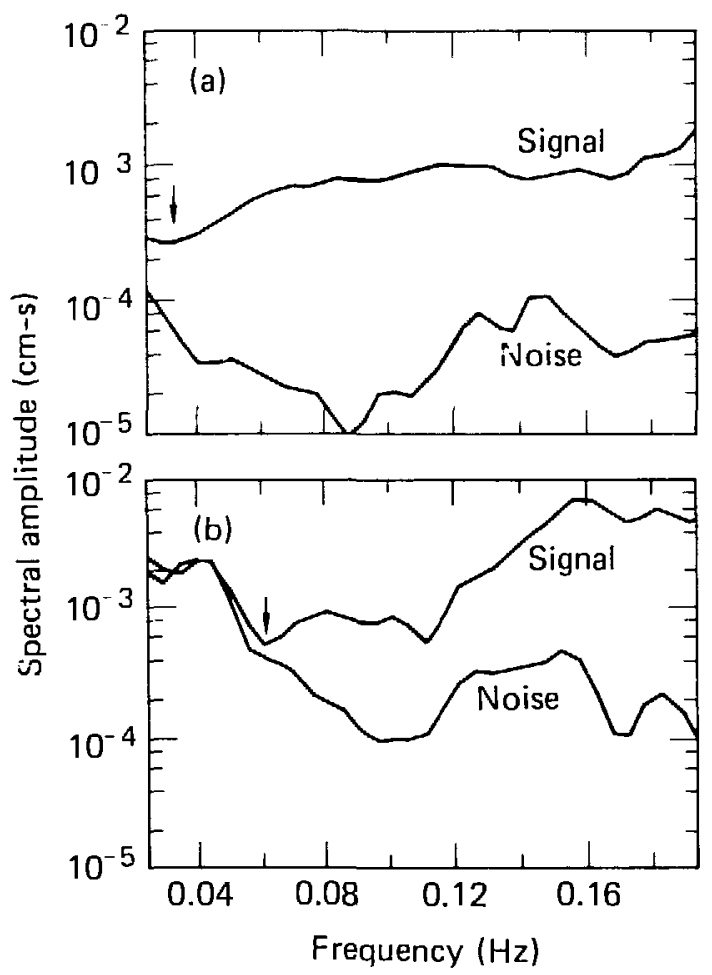

Figure 11. Displacement amplitude spectra of signal and noise time series. The arrow indicates the lowest Frequency with satisfactory sigual to noise ratio for (a) the vertical component of motion recorded at Mina for the Nessel explosion and (b) the transverse component of motion recorded at Kanab for the Pyramid explosion. 
surface waves for several events indicated in Table 1 could not be studied. The magnitude threshold for surface-wave studies is as high as $m_{b} 4.6$ during these high noise time periods.

SURFACE-WAVE SOURCE SPECTRA

In this section, we calculate source spectra using both the signal amplitude and phase spectra computed in the previous section. The amplitude and phase source spectra will be modeled for source paraneters in subsequent sections.

SOURCE AMPLITUDE SPECTRA

A Hanning operator was applied to smooth the amplitude spectra computed in the previous section. The smoothed amplitude spectra were normalized to a propagation distance of $500 \mathrm{~km}$ by multiplying by the factor $\sqrt{\mathrm{r} / 500}$, where $\mathrm{r}$ is the epicentral distance. No correction was made for attenuation of surface-wave amplitudes because we expect that the effect of attenuation is small for the short epicentral distances and long wavelengths in this study. For example, the surface-wave attenuation factor is $e^{-n r}$ where $n=\pi / Q U T$, $Q$ is quality factor, $U$ is group velocity, and $T$ is period. Our greatest epicentral distance is about $400 \mathrm{~km}$. Assuming a very low $Q$ of 50 , the attenuation factor is about 0.4 for a $10 \mathrm{~s}$ period wave traveling $3 \mathrm{~km} / \mathrm{s}$. This value of the attenuation factor represents an extreme because of the low $Q$ value and maximum distance. A more reasonable $Q$ value of 200 gives an attenuation factor of about 0.8. On the basis of these calculations, we argue that attenuation of surface wave amplitudes is not more than a factor of 2 for periods ( 6 to $25 \mathrm{~s}$ ) and distances ( 200 to $400 \mathrm{~km}$ ) in this study.

\section{SOURCE PHASE SPECTRA}

The surface-wave source phase, $\phi$, which is measured in cycles, was computed by the following equation:

$\phi^{s}=\phi^{0}+E t-\frac{E r}{C}$ 
whers

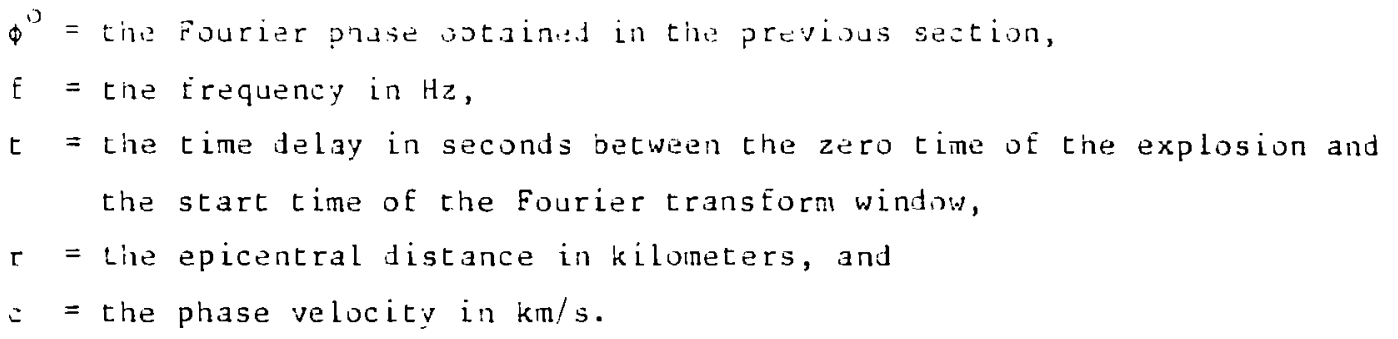

The term $f r / c$ is the phase delay due to propagation, which is subtracied from the phase delay equalized to the zero tine of the explosion, $\phi^{0}+f t$, to bytain the source phase. This calculation gives values of the far-field source phase relative to zero time of the explosion. The phase velocities of Love and Rayleigh waves were caken Eron the studies of Priestley and 3 rune ${ }^{9}$ and patton, 3 respectively.

\section{RESULTS}

line Rayleigh-wave source amplitude and phase spectra for Eive explosions are shown in Figs. 12 and 13, respectively.

The spectra for explosions Pyramid, Nessel, and burzet show more variability than do the spectra for explosions Hearts and offshore. Oor example, the phase spectra at Elko show signiticant phase shifts in the case of explosions Pyramid, Nessel, and Burzet. The amplitude spectra at Elko for the Pyramis explosion seens to be enriched in high Erequencies relative to the other stations. Kanab's amplitude spectrum at frequenries above $0.1 \mathrm{~Hz}$ has a peculiar dip, unlike the spectra at the other stations. This feature does not appear to change much Erom explosion to explosion.

An isotropic explosive source does not generate Love waves. However, Love waves were observed Eor all explosions. The Love-wave amplitude spectra are shown in Fig. 14. Many of the Love-wave amplitude spectra appear to be enriched in the higher frequencies. This is especially true for frequencies higher tinan 0.10. The enhancement of the higiner frequencies is most apparent for the Nessel and Pyranid explosions (Figs. lite and b, respectively).

The Love-wave phase spectra are shown in $\mathrm{Fig}$. 15. The error in the Lovewave pinase measurements is greater than that in the Rayleigh-wave measurements because the Love-wave phase velocities are not known as accurately as the Rayleigh-wave phase velocities. Greater uncertainties in the Love-wave phase velocity account for some of the wild variations in tne Love-wave phase. 

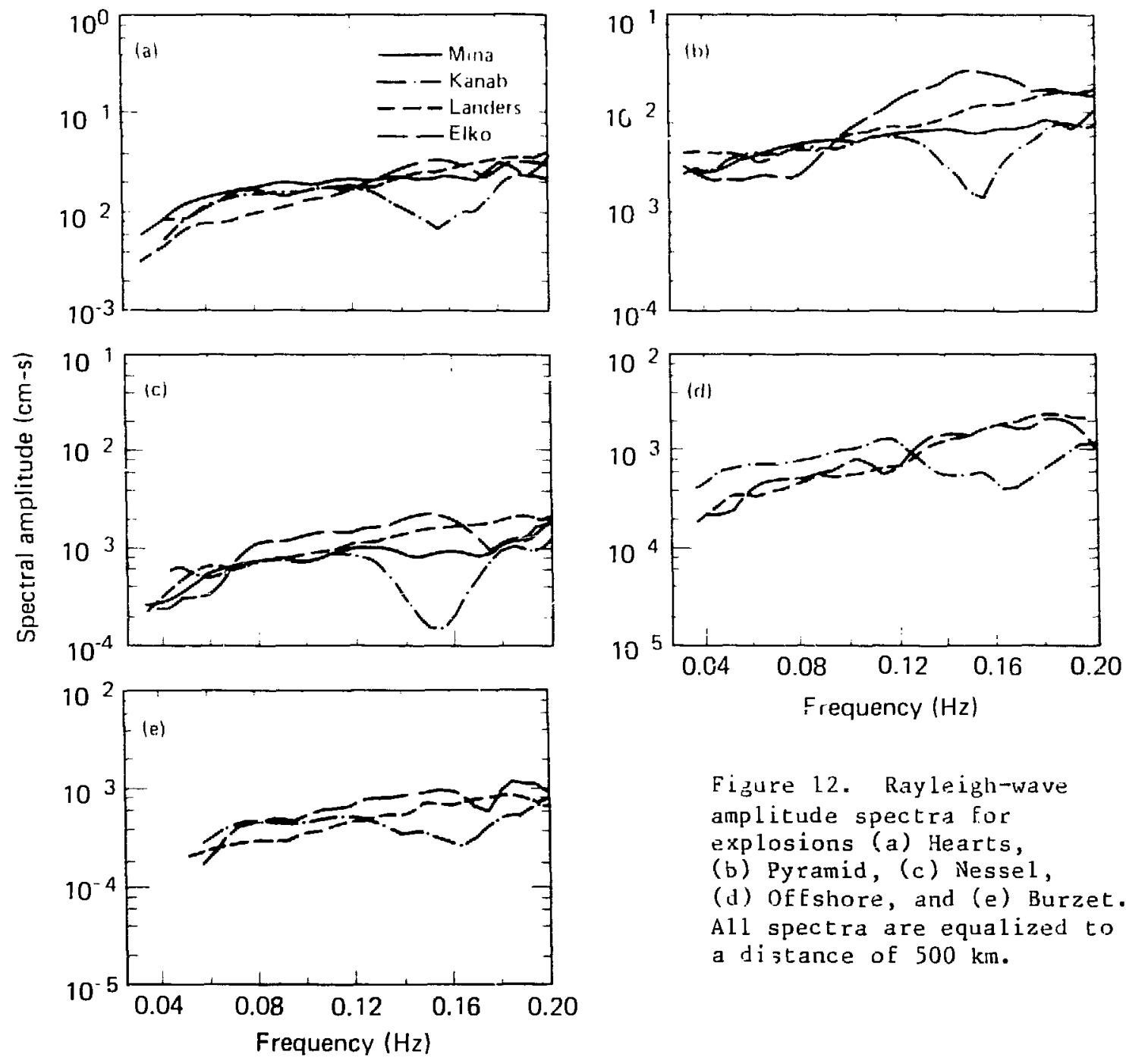

Fizure 12. Rayleigh-wave amplitude spectra for explosions (a) Hearts, (b) Pyramid, (c) Nessel, (d) Offshore, and (e) Burzet. All spectra are equalized to a distance of $500 \mathrm{~km}$. 

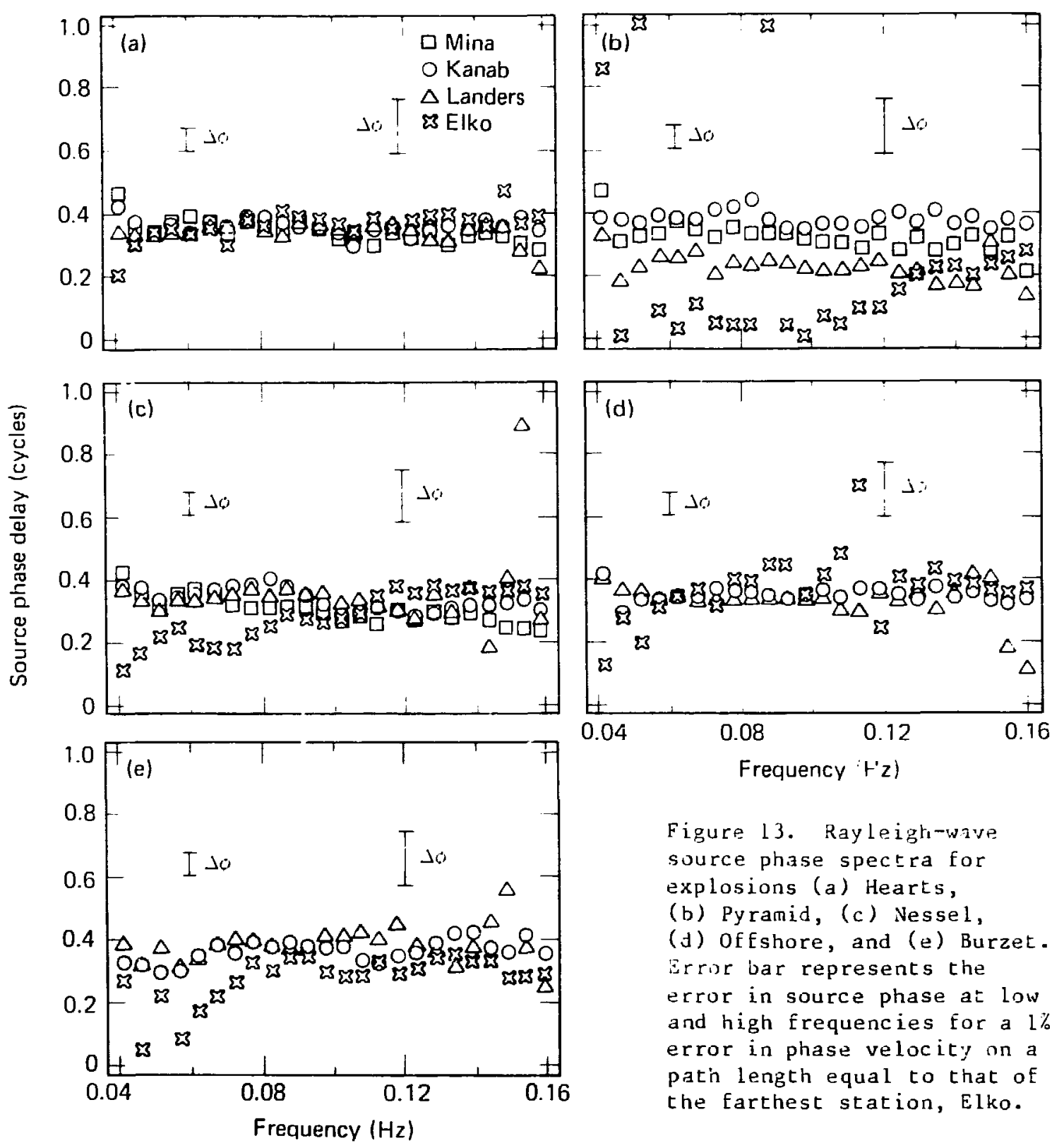

Figure 13. Rayleigh-wave source phase spectra for explosions (a) Hearts, (b) Pyramid, (c) Nessel, (d) offshore, and (e) Burzet. Error bar represents the error in source phase at low and high frequencies for a $1 \%$ error in phase velocity on a patin length equal to that of the farthest station, Elko. 

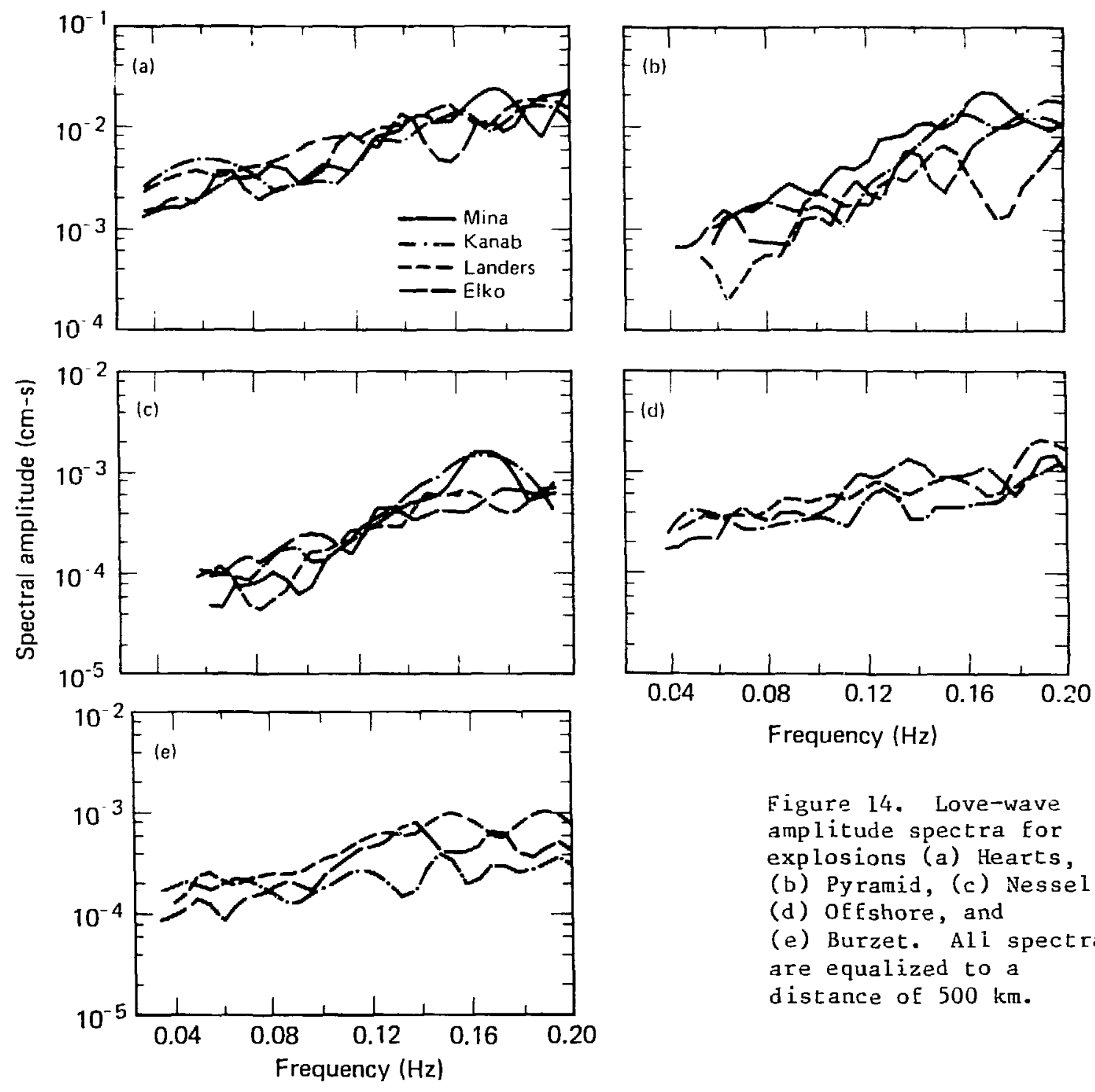

Frequency $(\mathrm{Hz})$

Figure 14. Love-wave amplitude spectra for explosions (a) Hearts, (b) Pyramid, (c) Nessel, (d) Offshore, and (e) Burzet. All spectra are equalized to a distance of $500 \mathrm{~km}$. 

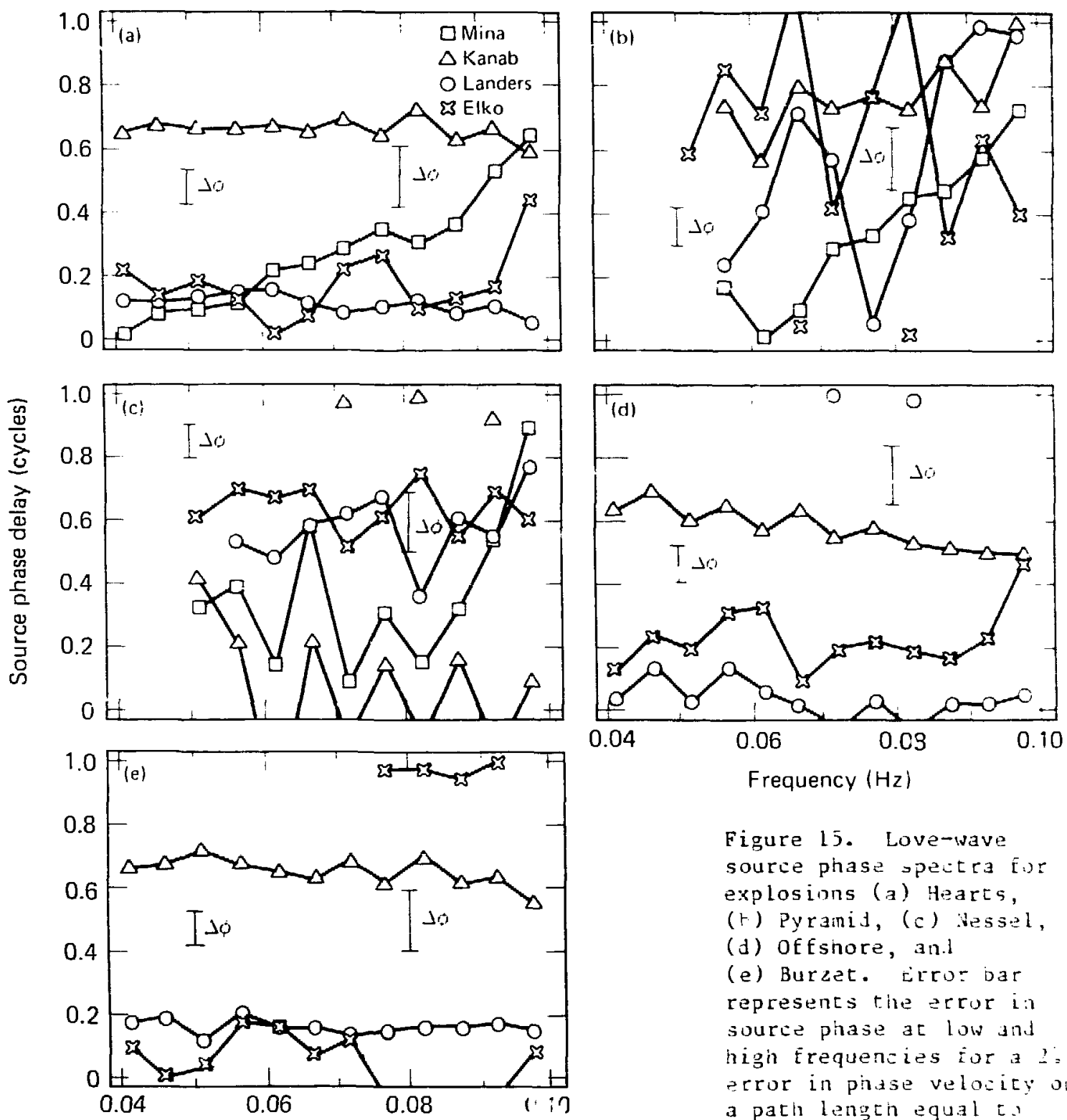

Frequency $(\mathrm{Hz})$

Figure 15. Love-wave source phase spettra for explosions (a) Heares, (r) Pyranit, (c) vessel,

(d) Offshore, an.l

(e) Burzat. Ėtro: dar represents the error $i: a$ source phase ar low and high Erequencies ior a 2 : error in phase velocicy on a path lengen equal $[$.

Frequency $(\mathrm{Hz})$ chat of the farthest station, Elks. 


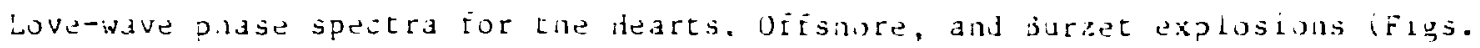

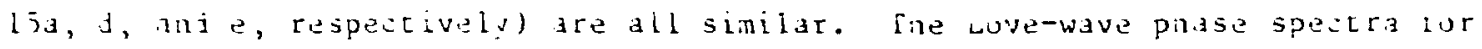
inesel (rig. los) appear to be sinifted oy a nalf cycle (i.e., $\pi$ ) relative to tide piase for the other events at each station except Mina. The piase spectr.s Eur Pyranid (Fig. Lij) are fificult to interpret.

YODELING THE ERLUSION SOURLE

Tire purpose of this section is co investizate the chasateristics oi ta. reiducej lisplacement potential tuat affect the generation of low-freyuenc: seismic waves. Ne will tian atteapt to molel the Rayleigitsave source specta

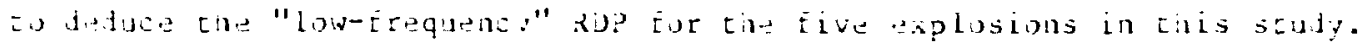

Fie seismiz soure due to the detonation of undergroud nuclear explosion vill be regarded as a point source because of the long wavelengta or our soservations. For a spherizally symmetric explosive source, Lovell outained a point source representation consisting of taree mutuall; perpendicuiar, sutward directed, dipole turces a $\overline{\mathrm{t}}$ equal strengtin. The seisinic nument

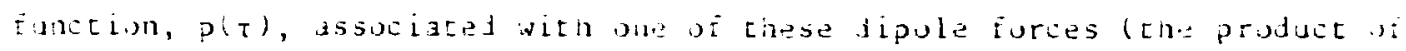

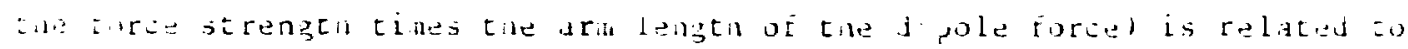
andim parimeters at tae source and tae reduced displazement putential,

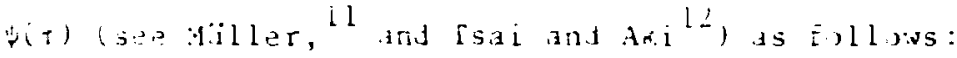

$P(\tau)=4 \pi \rho \alpha^{2} \psi(\tau)$,

where $p$ is the densicy, $a$ is compressional velocity, and $\tau$ is tine reduced travel time at a radius, $r$, from the center of the explosion. Th: radial displacement at this radius is written in terms of the RDP as

$D(r, \tau)=-\frac{\partial}{\partial r}\left[\frac{\psi(\tau)}{r}\right]$,

ia i base of an infinite nornogeneous elastiz nedium. The seismiz noment of the explosion succe, 1, , is the static level that the function $p(\tau)$ reaches a $t \rightarrow \infty$. lill $\mathrm{r}^{1}$ used the two equations above to relate tne monent ro the 
displacement, $D_{1}$, at the elastic sphere around the explosion and to the surface area, $s_{1}$, of the sphere

$$
I_{e}=(\lambda+2 \mu) S_{1} D_{1}
$$

where $\lambda$ and $\mu$ are elastic medium constants at the radius of the sphere.

The spectrum of Rayleigh waves in a vertically heterogeneous medium at a distance, $r$, from the explosion source may be written using the excitation formalism of Saito ${ }^{13}$ and the moment tensor representation

$u(r, \omega)=\frac{Y_{1}(0)}{4 \mathrm{CUI}} \sqrt{\frac{2}{\pi k r}}=-i\left(\frac{\omega r}{C}-\frac{3 \pi}{4}\right)$

$$
\left[\left(M_{x x}+M_{y y}\right) \frac{k Y_{3}(h)}{2}-M_{z z} \frac{Y_{2}(h)+\lambda(h) k Y_{3}(h)}{\lambda(h)+2 \mu(h)}\right]
$$

where ${ }^{\prime} \because:{ }_{y y}$, and $M_{z}$, represent the spectrum of the moment functions of each of the dipole forces. The Lamé parameters, $\mu$ and $\lambda$, are a function of depth only, where $h$ is the shot depth. The $Y_{i}$ are eigen functions of Rayleigh waves in a vertically heterogeneous medium, which satisfy the wave equations derived by Saito ${ }^{13}$ and Takeuchi and Saitn. ${ }^{4}$ For the spherically symmetric source described above, the spectrum of the moments may be written as

$M_{x x}(\omega)=M_{y y}(\omega)=M_{z z}(\omega)=P(\omega)=4 \pi \rho \alpha^{2} \Psi(\omega)$

where $P(\omega)$ and $\Psi(\omega)$ are the spectra of the functions, $P(\tau)$ and $\psi(\tau)$, respectively. It is clear from this mathematical description that the spectrum of the RDP is an essential source parameter controlling the surfase wave spectrum. The spectral characteristics of the RDP can de recovered firun the surface-wave spectrum, provided that the medium is accounted for by the mediun responses expressed in terms of the $Y_{i}$ in Ë. (5) and the propagation from source to receiver. 
Three ccmmonly suggested functional forms of the RDP are: (1) a step function (permanent displacement), (2) an impulse function (a delta function with no permanent displacement), and (3) a function with overshoot (i.e., an RDP with finite rise time, overshoot, and a permanent static displacement shich is less than the peak value of the overshoot). A mathematical representation of the function with overshoot (see Von Seggren and Blandford ${ }^{15}$, is

$\frac{\psi(t)}{\psi(\infty)}=\left\{1-e^{-K t}\left[1+K t-B(K t)^{2}\right]\right\} H(t)$

where $K$ controls the rise $t$ ime and width of the overshoot, $B$ controls the amount of overshoot, and $H(t)$ is the Heaviside function. A step Eunction is obtained by making $K$ very large and $B=0$; i.e., $K=1000, B=0$. The impulse function cannot be represented by this formula because the behavior of the function at large $t$ is a constant of value 1 . The amplitude scale factor, $\psi(\infty)$, is defined at large times and determines the permanent static displacement on the elastic sphere around the explosion point. The permanent static displacement is directly proportional to the explosion moment, as shown in Eq. (4).

\section{PARAMETER STUDY}

In order to examine the effects of the RDP on the surface-wave source spectrum, a parameter study was carried out involving the mathematical Eunctions described above. The frequency dependence of $P(\omega)$ was determinal by the spectrum of the RDP time function. The amplitude scale factor was set to give a seismic moment of $10^{25} \mathrm{~d} y \mathrm{n}-\mathrm{cm}$ in every case. For the impulse, the scale factor corresponds to the peak strength of the dipole force syctem. The medium model adopted to calculate $Y_{i}$ is the Gutenoerg continental earth model (for medium parameters see [sai and Aki ${ }^{l}$ ).

The results of the parameter study are shown in Fig. lo. The Rayleighwave amplitude and phase spectra in the frequency range, 0.03 to $0.167 \mathrm{~Hz}$ are siown for the different RDP functions. The extremes are represented oy the impulse and step functions. The Von Seggren-Blandford time functions show 


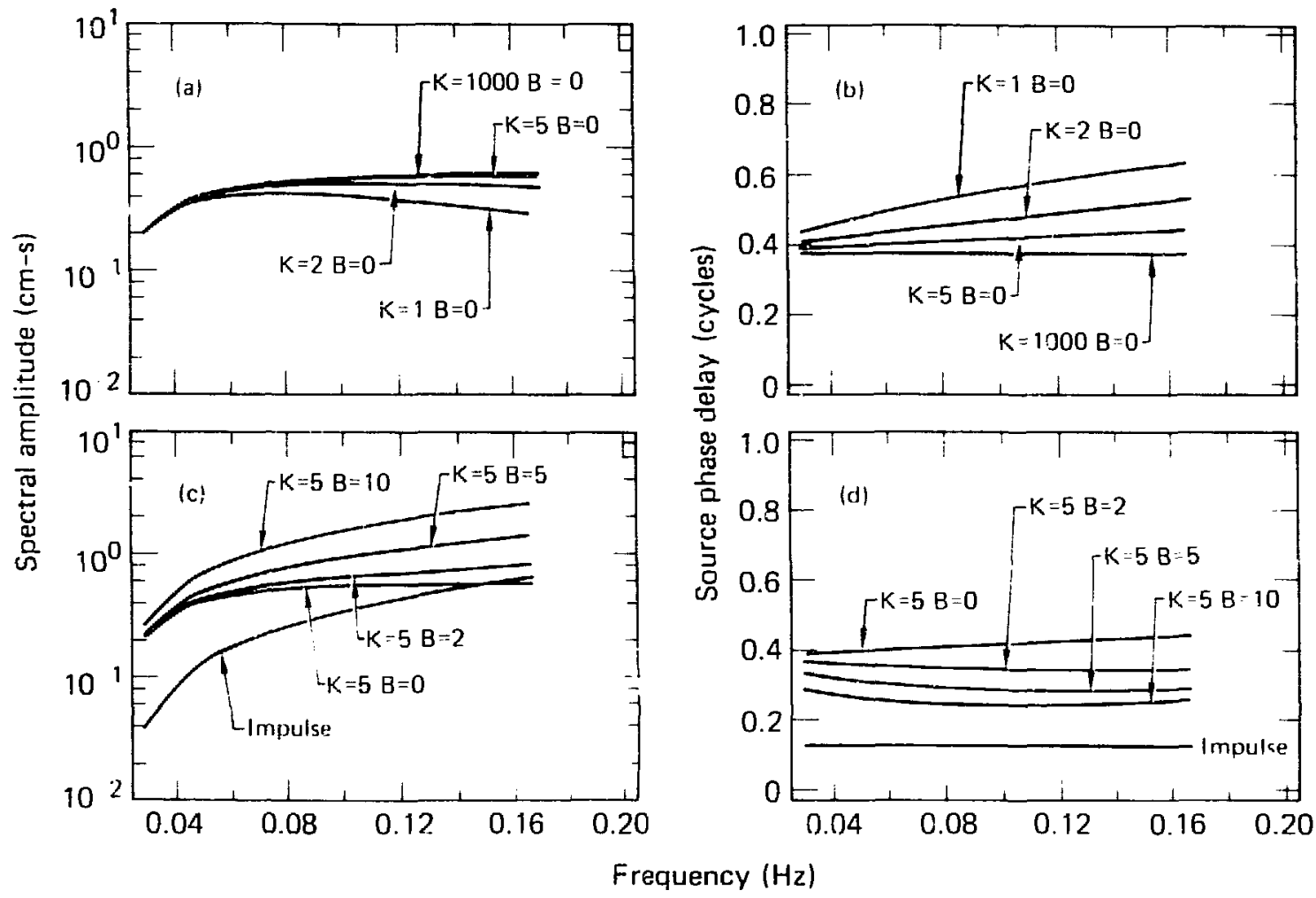

Figure 16. The effect of $K$ and $B$ (the risetime and overshoot paraneters in Von Seggren and Blandford's mathematical representation of the RDP 15 ) on Rayleigh-wave spectra. The parameter, B, is held constant in (a) amplitude spectra and (b) phase spectra. The parameter, $K$, is held constant in (c) amplitude spectra and (d) phase spectra. 
some characteristics of the impulse and step functions depending on the Erequency and the parameter values. Specifically, when the risetime is held conscant and overshoor is varied, the shape of the amplitude spectrum for the case $K=5$ and $B=10$ is very close to the shape of the spectrum for an inpulse. The phase spectrum for tinis case is delayed about 0.15 cycles nore than an impulse. If we went to high enough $B$, both the amplitude and tne phase spectra would be very glose to that of the impulse over this limited Erequency range. When overshoot is held constant and risetime is varied, the effect on the Rayleigh-wave spectruin is less than the efiect due to oversioot. The risetime is expected to scale with the cube-root of the yield of the explosion (see carpenter et al. ${ }^{17}$ ). For the explosions in tis study, $k$ should certainly be greater than two. On the basis of results in Fig. Lo, we can safely dismiss risetime as a critical paraneter in modeling tine long period surface-wave spectra.

\section{MODELING RESULTS}

The results of the paraneter study indicate that some of the variability CE the observed Rayleigh-wave spectra could be caused oy differences in the RDP irom shot to shot. There is experimental evidence for dependence of tre ROP on the shot medium (see Werta and Herbst ${ }^{18}$ ). Furthermore, For a given explosion there may be directional dependence of the RDP. For exanple, horizontal traveling P-waves appear to have a different Rup thai downivad traveling P-waves (see Blandford ${ }^{19}$ ). There could be an azimuthal dependence of tne RDP due perhaps to a anisotropy of the medium's physical properties. In this section, all of the variability seen in the observed Rayleigh-wave spectra is assumed to arise from the RDP alone. In order to match the observed spectra, it will be necessary to invoke azimuthal dependence. Since other source effects may be responsible for introducing asymmetries into the seismic wave radiation and since, furthermore the RDP concept is derived from a spherically symmetric model, it may be appropriate to call the function obtained from the modeling an "apparent" RDP.

The trial and error fit is made to just the observed amplitude spectrum. Only the overshoot parameter, $B$, was varied, with the risetime, $1 / K$, Fixed at a value of 0.2 . The seismic moment, $M_{e}$, was determined by scaling tine 
theoretical spectrum to each observed spectrum individually. Examples of the comparisons between theoretical and observed spectra are shown in Fig. 17 and the results are summarized in Table 4.

There are several noteworthy results in Table 4. First, there appear to be small differences in the values of the parameter $B$ between events. The differences between stations are more noticeable than the differences between explosions. We note that Elko and Landers consistently "see" a source with more overshoot than do Mina or Kanab. This is most apparent in the amplitude (and phase) spectra of Rayleigh waves recorded at Elko for events Nessel and Pyranid.

\section{EFFECT OF CRUSTAL STRUCTURE}

Hudson and Douglas ${ }^{20}$ investigated the effect of shallow crustal structure on the excitation of Rayleigh waves by explosion sources and showed that the Rayleigh-wave excitation by explosions in an alluvium crust is greater than the excitation by explosions in a granitic crust, particulariy at Erequencies above $0.1 \mathrm{~Hz}$ (see Fig. 1 of Ref. 20). At low Erequencies, the effect of shallow crustal structure on Rayleigh-wave excitation is small. Nevertheless, it is possible that the frequency dependence of the Rayleighwave amplitudes attributed to RDP is an artifact of the earth model assumed in

mible 4. Apparent reduced displacement potential.

\begin{tabular}{|c|c|c|c|c|}
\hline \multirow{3}{*}{$\frac{\text { Stations }}{\text { Mina }}$} & \multicolumn{4}{|c|}{ Explosions } \\
\hline & & Hearts & Nessel & Pyramid \\
\hline & & $\begin{array}{l}=2.0 \\
=2.9 \times 10^{23}\end{array}$ & $\begin{array}{l}B=2 \text { to } 5 \\
M_{e}=8 \times 10^{21}\end{array}$ & $\begin{array}{l}\mathrm{B}=2.0 \\
\mathrm{M}_{\mathrm{e}}=7.5 \times 10^{22}\end{array}$ \\
\hline Kanab & & $\begin{array}{l}=2.0 \\
=2.5 \times 10^{23}\end{array}$ & $\begin{array}{l}B=2 \text { to } 5 \\
M_{e}=7.4 \times 10^{21}\end{array}$ & $\begin{aligned} \mathrm{B} & =2.0 \\
\mathrm{M}_{\mathrm{e}} & =7.8 \times 10^{22}\end{aligned}$ \\
\hline Landers & & $\begin{array}{l}=5 \text { to } 10 \\
=9 \times 10^{22}\end{array}$ & $\begin{array}{l}B=5.0 \\
M_{e}=9 \times 10^{2 l}\end{array}$ & $\begin{array}{l}B=5.0 \\
M_{e}=4.7 \times 10^{22}\end{array}$ \\
\hline Elko & & $\begin{array}{l}=5.0 \\
=1.9 \times 10^{23}\end{array}$ & $\begin{array}{l}B=10.0 \text { or impulse } \\
M_{2}=7.4 \times 10^{21}\end{array}$ & $\begin{array}{l}B=10.0 \text { or impulse } \\
M_{e}=4.9 \times 10^{22}\end{array}$ \\
\hline
\end{tabular}




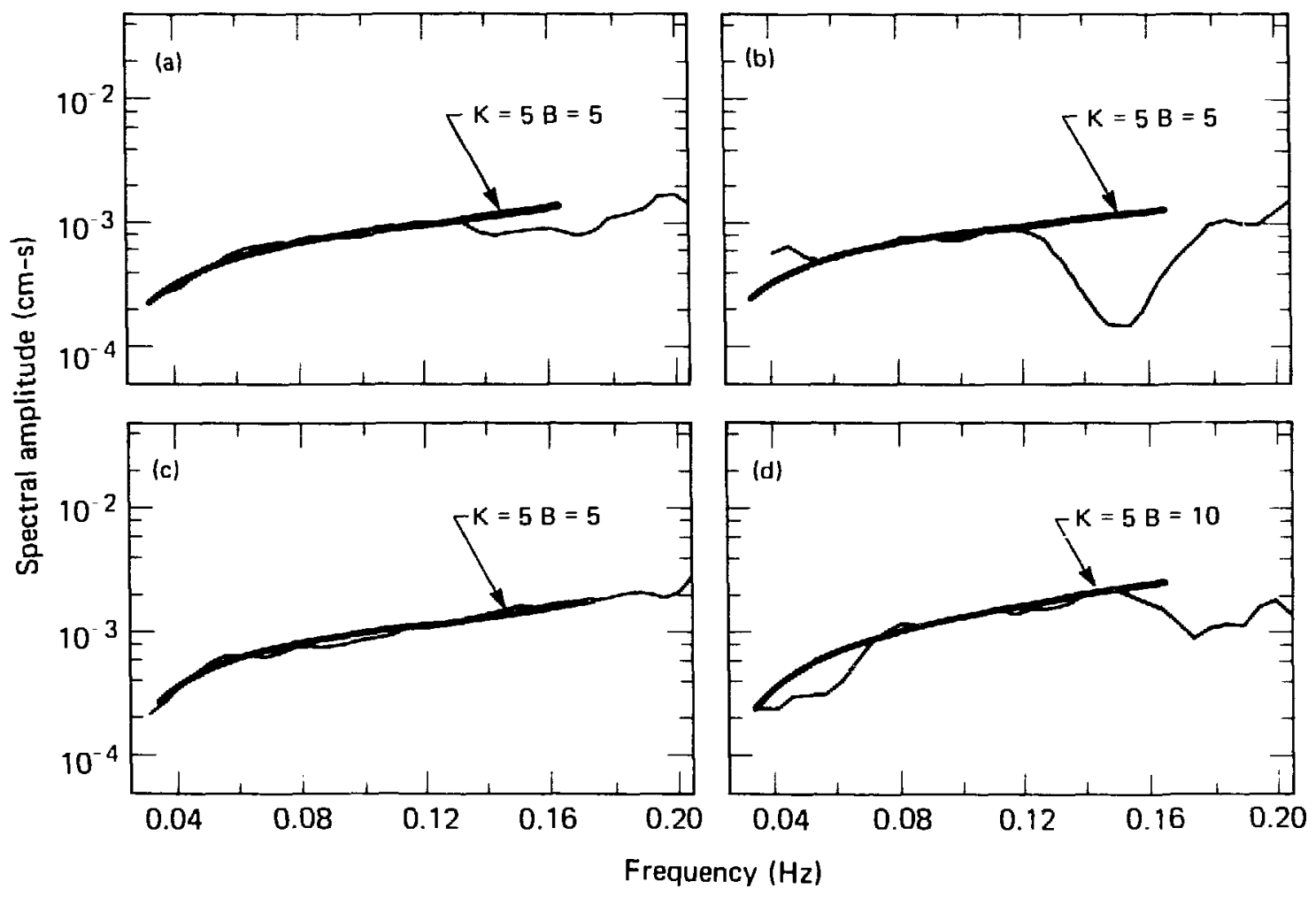

Figure 17. Comparison of observed Rayleigh wave amplitude spectra with theoretical spectra obtained by adjusting the parameters $B$ and $\mathrm{M}_{e}$ (see text) for each station individually. The explosion is Nessel. 
the calculation of the theoretical spectra. In order to check this, we examined an alternative earth model.

The parameters of a model appropriate for the Basin and Range Province of western U.S. are given in Table 5. The upper mantle velocities and densities are taken from the study of Priestley and Brune. ${ }^{9}$ The crustal P-wave velocities are based on the resules of Prodehl 21 Eor a refraction line from NTS to Elko. The cruscal S-wave velocities were chosen to agree with the Rayleigh-wave phase velocity dispersion between NTS and Elko (see Patcon ${ }^{8}$ ). The Rayleign-wave spectra for explosions in this velocity model are compared to the spectra in the Gutenberg model in Fig. 18.

The comparison in Fig. 18 indicates that the amplitude excitation by explosions in the new velocity model is significantly greater than the excitation in the Gutenberg model at high frequencies. Indeed, the amplitude spectrum for a source with overshoot $(B=5)$ in the Gutenberg model is very similar to the spectrum for a step-function source in the Basin and Range velocity model. The Rayleigh-wave phase spectrum for an explosion source is not affected by the crustal structure in the frequency range of interest.

Table 5. Basin and Range velocity structure.

\begin{tabular}{cccc}
$\begin{array}{c}\text { Layer } \\
\text { thickness } \\
(\mathrm{kin})\end{array}$ & $\begin{array}{c}\text { P-wave } \\
\text { velocity } \\
(\mathrm{kin} / \mathrm{s})\end{array}$ & $\begin{array}{c}\text { j-wave } \\
\text { velocity } \\
(\mathrm{km} / \mathrm{s})\end{array}$ & $\begin{array}{r}\text { Density } \\
(\mathrm{g} / \mathrm{cc})\end{array}$ \\
\hline 1.25 & 3.20 & 1.88 & 2.20 \\
20.75 & 6.00 & 3.46 & 2.78 \\
10.00 & 6.80 & 3.87 & 2.96 \\
29.00 & 7.80 & 4.50 & 3.30 \\
40.00 & 7.80 & 4.12 & 3.30 \\
15.00 & 7.81 & 4.10 & 3.40 \\
20.00 & 7.85 & 4.05 & 3.40 \\
20.00 & 7.87 & 4.05 & 3.42 \\
20.00 & 7.92 & 4.06 & 3.44 \\
\hline
\end{tabular}


The velocity model in Table 5 is probably a good approximation of the best plane-layered model to represent the medium between NTS and the Livermore stations (with the possible exception of Mina). Therefore, on the basis of the results in Table 4 and the comparisons in Fig. 18, we conclude that little or no overshoot is required to model the shape of observed Rayleigh-wave amplitude spectra below $0.16 \mathrm{~Hz}$ for any explosion, including Burzet and offshore. What overshoot is present in the RDP appears to be greater for azimuths nortin and south of NTS (Elko and Landers) than for azimuths east and west (Mina and Kanab).

It was argued in the section on Surface-Wave Source Spectra that the effect of attenuation on the spectra was small enough to igriore because of the short path lengths. Nevertheless, attenuation might be great enough to cancel the effects of overshoot at the highest frequencies where our modeling is particularly sensitive. We considered this possibility but found two lines of evidence against it. One is the fact that the observed amplitude spectra for the closer stations (Mina and Kanab) were modeled by an RDP with less overshoot than the spectra observed at Landers or Elko which have greater epicentral distances. The second evidence is the Rayleigh wave source phase in Fig. 13. Although the error at high frequency on each data point is too large to discriminate between overshoot or no overshoot, on average the observed phase spectra seem to be consistent with small (B $\sim 2$ ) overshoot. The phase spectra at Elko For explosions Pyramid, Nessel, and Burzet are notable exceptions.

The fact that the Rayleigh-wave radiation is asymetric and shows variability from explosion to explosion is not completely surprising, since Love waves, an indicator of secondary sources or source asymmetry, were also recorded. However, the amplitudes of the long-period Love waves are quite small compared to the Rayleigh waves. It is questionable whether the Lovewave source generates Rayleigh waves large enough to perturb the explosion's isotropic radiation in the fashion and to the degree that we observe. We will address this question and the modeling of the Love waves in the next section. 


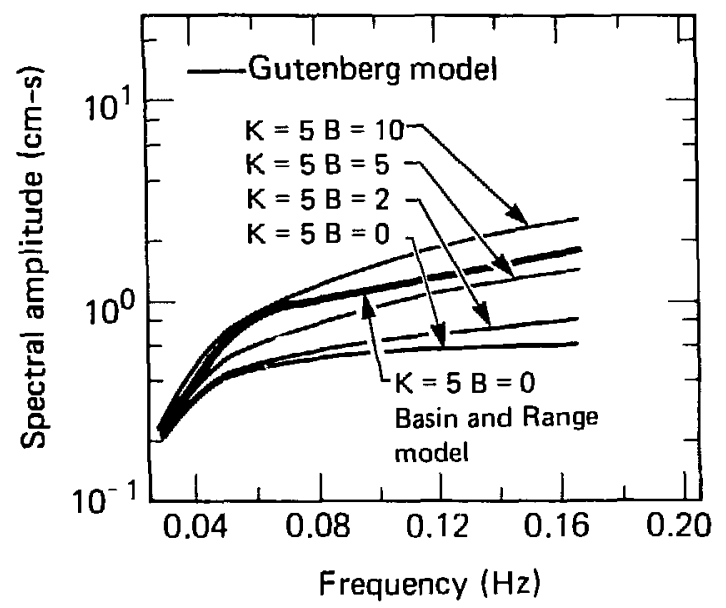

Figure 18. Comparison of Rayleigh-wave spectra calculated for the Basin and Range velocity model in Table 5 and for the Gutenberg velocity model. Notice that the shape of the spectrum calculated for the Basin and Range model using a step function resembles the shape of the spectrum with overshoot $(B=5)$ of the Gutenberg model. 
In addition to the explosion source, there are secondary sources of seismic waves caused by the interaction of the shock wave with the surrounding medium. Secondary sources are known to contribute significantly to the seismic wave field of an underground explosion, as evidenced by the commonly observed shear waves $(\mathrm{SH})$ and Love waves on the seismograms of these events. Indeed, a significant proportion of the seismic wave field at low frequencies can be excited by the secondary source(s). In this section we explore secondary sources that could be dominating the generation of low-frequency seismic waves. These sources are: spall slapdown, shown by Viecelli ${ }^{22}$ to be an efficient Rayleigh-wave source; thrust faulting model of Massé, 3 and tectonic strain release by earthquake triggering (see Brune and pomeroy, 23 Aki, ${ }^{24}$ Aki et al., ${ }^{25}$ and Aki and Tsai ${ }^{26}$ ).

SPALL CLOSURE

Viecelli ${ }^{22}$ describes spallation as the parting of horizontal layers of rocks in the earth at a depth where the tensile stress of the shock wave reflected off the free surface exceeds the tensile strength of the medium. The spalled layers experience ballistic flight and eventually free-fall back to the earth's surface. The impact of the spalled layers on the surface of the earth is termed spall closure and has been shown to be an efficient means of exciting Rayleigh waves (see Viecelli ${ }^{22}$ ). In this section we explore briefly the possibility that the long-period Rayleigh waves were generated by this secondary source.

In the formulation of a mathematical source representation, the spallation event (i.e., the parting of layers in tension) is usually dismissed as being a small or inefficient source of seismic waves compared to the spall closure event. The body-force representation of spall closure is a downwarddirected force distribution, normal to the surface. For long wavelengths this 
distribution is reduced to a single point force with impulse, I, equal to (viecelli $i^{22}$ )

$$
I=\frac{\pi}{3} \rho d_{0} r_{0}^{2}\left(2 h_{0}\right)^{1 / 2}
$$

where

$$
\begin{aligned}
& r_{0}=\text { the radius of a spalled plate of material with density } p, \\
& d_{0}=\text { the thickness of the plate, } \\
& h_{0}=\text { the maximum height it atcains, and } \\
& g=\text { acceleration of gravity. }
\end{aligned}
$$

In a homogeneous half space, an impulsive downward point force generates Rayleigh waves with the same shape amplitude spectrum as an explosive source with step function time dependence (see Aki et al. ${ }^{27}$ ). The differenc. is that the downward force generates Rryleigh waves which are delayed by $\pi / 2$ radians at all frequencies relative to the explosion's Rayleigh waves. These characteristics of Rayleigh waves in a homogeneous half space also hold true in a layered earth structure at least in the frequency range of interest in this study.

Since spallation and spall closure are comnonly observed on near-field strong motion accelerographs both for past explosions (see Eisler et al. ${ }^{28}$ ) and for the recent explosions, including some of the shots in this study (Warshaw ${ }^{29}$ ), the question arises whether spall closure generaces significant amounts of long-period seismic energy that reach the far-field. Comparisons between observed Rayleigh-wave spectra for the Nessel explosion and theoretical spectra based on the model of spall closure described above are shown in Fig. 19. The impulse obtained from the fit of the theoretical to the observed amplitude spectra is $1.3 \times 10^{16}$ dyn-s which is in good agreenint with the value obtained from the yield-impulse relationship reported by viecelli. 22 (For Hearts and Pyramid, the observed impulse was about a factor of 3 greater than the predicted value.) However, the theoretical source phase for the model of spall closure does not match the observations. The observed phase values are significantly less than the predicted valies. Consequently, the source phase observations indicate that spall closure as proposed by Viecelli22 is not the source of long period Rayleigh waves in the far-field. 

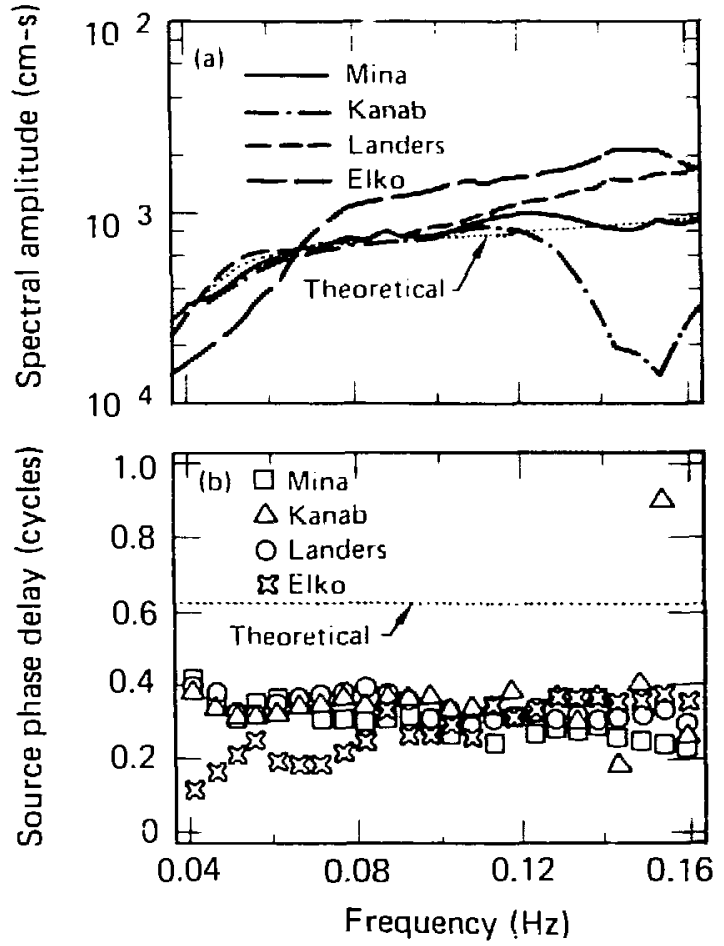

Figure 19. Comparison of observed Rayleigh-wave amplitude and phase spectra for Nessel explosior with the calculated spectra for spall closure model. 
It may be that the explosion and spall closure sources combine to generate the long-period waves as proposed by Bache et al. 30 and Peppin. 31 However, a significant contribution by spall closure is not supported by our phase observations. A multiple source model with significant spall component also predicts source-phase delays greater than 0.375 cycles, which is not observed in any of the spectra. Furthermore, the asymmetries in Rayleigh-wave radiation patterns and the Love-waves cannot be modeled by the addition of spall closure since it radiates isotropically. We concur with the conclusions reacined by Bache et al. ${ }^{30}$ that spall closure probably does not contribute great ly to the generation of long-period waves recorded in the far-field, at least for the Yucca Valley explosions in this study.

\section{THRUSI FAULTING}

Masse ${ }^{3}$ has recently proposed a new source model which has four "stages" corresponding to various sources of seismic radiation for underground uclear explosions. The first stage is the explosion itself. The other stages are all secondary sources, the third stage being spall closure which was discussed in the previous section. The second staje is induced tinust faulting along preexisting joints and fracture planes close to the source. Rather than a tectonic release, Massé envisions a passive sliding on planes of weakness with the passage of the shock wave from the explosion. Support for this type of faulting at NTS comes from observational studies of seismic waves reviewed by Massé and from field observations of vertical movement on faults in the source region after underground explosions (see McKeown and Dickey ${ }^{32}$ ). In the Massé source model, thrust faulting is the only source that generates SH waves and Love waves.

The thrust Fauling believed to be induced is shown in detail in Fig. 20 , reproduced From Massé. 3 The pattern of faulcing is not spherically symmetric about ground-zero, as may be seen in this figure. Ratier, the strikes of the faults tend to follow the "geologic fabric" of the source medium. According to Massé, the dip angle of the faulcs are commonly found to be close to $45^{\circ}$.

The problem is to formulate a nathematical source representation for tae geometry of thrust faults shown in Fig. 20. Tnis representation can be yuite easily formulaced for a slip dislocation on planar ialles. ide know from 


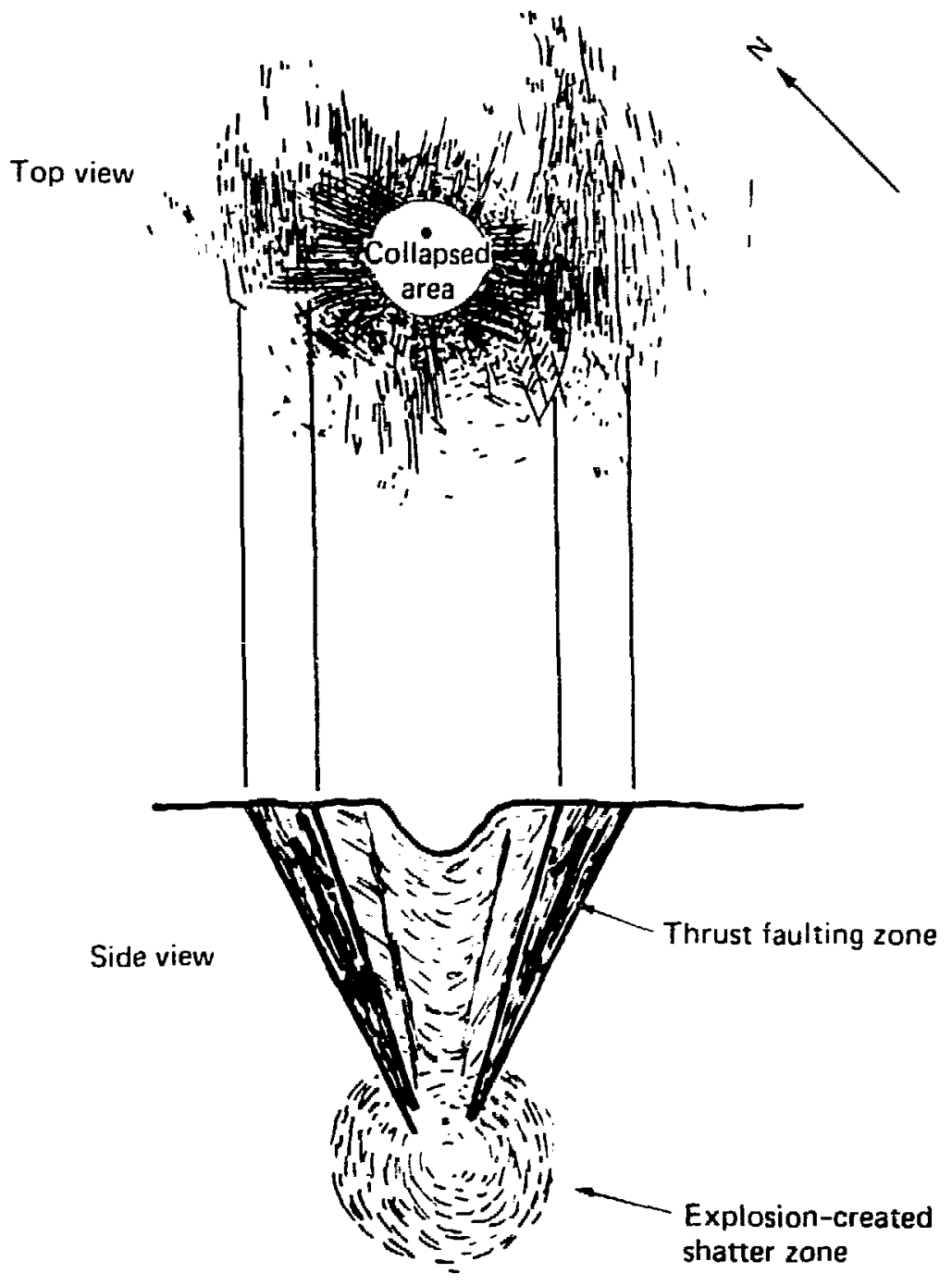

Figure 20. Top view shows surface fracture patcern proúced above an explosion deconaced in Yucca Valley. Side view shows theorized thrust faults produced by the explosion's compressional stress wave. This Eigure is reproduced Eron a paper by Massé. 3 
- Lastudynamies chat a tisplacenent discontinuly for a puint source is equivalent to a point jouble-souple Eurze system navine no net anguiar moment. The orientation of the double-couple in the case of chrust $\bar{r} a u l t s$ is such that one couple acts alung the dip f the fault and the second acts at right angles to the fauit, canceling the anjular nonent of the first couple. If we consider $45^{\circ}$ dipping faults, the orientation of the dauble-couple is identical for any of the thrust faults in Fig. 20 whether chey dip to the nurch or co the south. Hence, for long wavelength seisnic waves, all of tio. tinrust faulcs can be replaced by a single double-couple benaving as a step function in tine. The seismic roment of this couple wibl consticute the sum w the noments of all the thrust faults that slipped with the passage of the S1700x wh Ve.

ic explured the possioility that thrust faulting is a secondary source ui long-period seisinic waves cinrough che Eolluwing mechod. First, the strike ot the thrust faults and the total seisinic noment wele deterinined oy phase and amplitude spectra ei the Love waves, respectively. Since taule strika and mionent are the only free parameters in che secondary source détined above, ind Love-vave data alone will constrain the secondary source representation for each explosion. jecond, we superimposed the cinrust source on the explosion (soe $p_{a c t o n}^{33}$ ) and adjusted the ratio of the strength of che carust source to the strength of the explosion in order to ininimize tine difference betwees voistred and calculated Rayleigh-wave spectra. The ratw oi soures strengtas is cne $F$ parameter used in the scudy by Toksöz and Keiner. 3+ Riller 1 obtained the relationsinip between $F$ and the seismic monents both of the explosion, $\mathrm{A}_{e}$, and of the earthquake, "Aq"

$F=\frac{x^{2}}{2 B^{2}} \frac{M}{x}$

idilere $\alpha$ and $B$ are $P$ - and S-wave velocities in che source region, respectively. Once the ratio $F$ is deterinined, the seismiz monent ot th. explosion nay be calculated using Eq. 11 .

File paramecers potained irom tha data fit are given in lable 0 . Fiase paraneters are che seismic moment and fault strike uf the secondary source lin this case a thrust fault), the ratio of source stiengtos, f, and the selsini: 
[asle J. Paranecers of secondary sumbe models obtained From lata fit.

\begin{tabular}{|c|c|c|c|c|c|c|}
\hline Explosion & $\begin{array}{l}\text { Source } \\
\text { model }\end{array}$ & $\begin{array}{c}y_{q}^{b} \\
(\text { Jyn-cm) }\end{array}$ & $\begin{array}{l}\text { Fault } \\
\text { strike } \\
\left({ }^{\circ}\right)\end{array}$ & $F$ & $(d y n-c m)$ & $2: 13^{c}$ \\
\hline Hearts & $\begin{array}{l}\text { SS } \\
\text { DS }\end{array}$ & $\begin{array}{l}3.1 \times 10^{22} \\
6.2 \times 10^{22}\end{array}$ & $\begin{array}{l}340 \\
295\end{array}$ & $\begin{array}{l}0.3 \\
0.5\end{array}$ & $\begin{array}{l}1.5 \cdot 10^{23} \\
1.9 \cdot 10^{23}\end{array}$ & $\begin{array}{l}0.11 \\
0.13\end{array}$ \\
\hline Pyranid & $\begin{array}{l}\text { SS } \\
\text { DS }\end{array}$ & $\begin{array}{l}0.6 \times 10^{21} \\
1.3 \times 10^{22}\end{array}$ & $\begin{array}{l}3+0 \\
295\end{array}$ & $\begin{array}{l}0.2 \\
0.3\end{array}$ & $\begin{array}{l}4.9 \cdot 10^{22} \\
0.5 \cdot 10^{22}\end{array}$ & $\begin{array}{l}0.17 \\
1) .16\end{array}$ \\
\hline UEEShore & $\begin{array}{l}\text { is } \\
\text { Ds }\end{array}$ & $\begin{array}{l}4.1 \times 10^{21} \\
3.1 \times 10^{21}\end{array}$ & $\begin{array}{l}340 \\
295\end{array}$ & $\begin{array}{l}0.7 \\
0.3\end{array}$ & $\begin{array}{l}0.8 \cdot 10^{2}-1 \\
1.5 \cdot 10^{2} ?\end{array}$ & $\begin{array}{l}0.111 \\
0.15\end{array}$ \\
\hline : Vessel & $\begin{array}{l}\text { SS } \\
\text { DS }\end{array}$ & $\begin{array}{l}9.3 \times 10^{20} \\
1.9 \times 10^{21}\end{array}$ & $\begin{array}{l}300 \\
255\end{array}$ & $\begin{array}{l}0.2 \\
0.3\end{array}$ & $\begin{array}{l}7.0 \cdot 10^{21} \\
9.5 \cdot 10^{21}\end{array}$ & $\begin{array}{l}0.13 \\
0.11\end{array}$ \\
\hline Burzet & $\begin{array}{l}\text { SS } \\
\text { DS }\end{array}$ & $\begin{array}{l}2.2 \times 10^{2} \mathrm{i} \\
4.4 \times 10^{21}\end{array}$ & $\begin{array}{l}340 \\
295\end{array}$ & $\begin{array}{l}0.5 \\
0.3\end{array}$ & $\begin{array}{l}5.5 \cdot 10^{2 !} \\
6.2 \cdot 10^{21}\end{array}$ & $\begin{array}{l}0.15 \\
0.14\end{array}$ \\
\hline
\end{tabular}

${ }^{3}$ SS $=$ strike-slip model (see roksöz and kehrer ${ }^{3+}$ ).

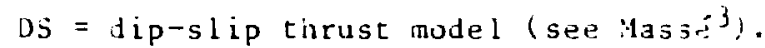

2 Erom love waves, Eaule strike measured from norch, posirive clockwise.

Root mean squared of differences between observed and calculated

Rayleigh-wave logarithm amplitude spectra for ali stations.

mument of the explosion. Also given in this table is the RMS error between observed and calculated amplicude spectra. This error reflects tire quality of fit to the Rayleigh-wave amplitude data for all stations.

Before discussing the results in rable 6 , we consider another model, tine strike-siip model of Toksöz and Kehrer.

TECTONLL STRALN RELEASE

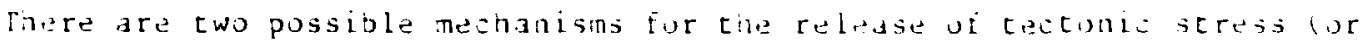
strain) oy an underground explosion. One nechanism is the formation of a region bi crushed rocks around the snot point witn essentiall: zero shear strength. The surrounding mediun, winch is under preexisting deviatori. stress, relaxes ditn che fornation of a zero-stress a avity. lnis is tae stress relaxation nodel (see press and drchambeas and drchamoeau and jamis $\left.{ }^{3 \prime \prime}\right)$. The otner mechanisin ialls for che shock wave to interact witn 
structural inhomogeneities; e.g., a nearby fault. The transient stress wave is great enough to overcome frictional stress on the fault and criggers an eartinquake. This is the earthquake-trigger model (see Brune and Pomeroy, 23 and $\mathrm{Aki}^{24}$ ). We shall adopt the earthquake-trigger model in this study and attempt to interpret our observations using the strike-slip faulr model proposed by Toksöz and Kehrer ${ }^{34}$ for NTS.

In the model of Toksöz and Kehrer, ${ }^{34}$ underground explosions release tectonic stress by strike-slip faulting on prominent geologic faults and explosion-produced faults aligned with the geologic fabric in the source region. Figure 2l, which is reproduced from Toksöz and Kehrer, shows the Eault-strike orientations obtained Erom the analysis of surface waves for explosions in Yucca Valley. There is generally good agreement between these orientations and the north-south geologic Eabric of the Yucca Valley. A right-lateral motion on the faults was assumed by Toksöz and keinrer in order to be consistent with the regional rectonic stress field, which shows generally northwest-southeast extension; e.g., Zoback and Zoback. 37

Because slip on faults is an efficient source of shear waves, the effect of si-ess release by underground explosions on surface waves can be quite significant, as shown by the calculations in Figs. 22 and 23 . Thes calculations pertain to an earthquake, modeled by a point double-couple source with step-function time dependence, superimposed on an explosion which also has step-function time dependence. The orientation of the double-couple is appropriate for a right lateral strike-slip fault with fault strike $22^{\circ}$ west of north. Figures 22 and 23 show the dependence of Rayleigh-wave spectra on F, the ratio of source strengths defined earlier, and on the depth of the double-couple. The Rayleigh-wave spectra are complicated because of the interaction of waves Erom two sources with different source phases. The effect of the interaction is greater in one azimuth, Elko, than it is in the other, Mina. At Elko, it is apparent that the efEect is smaller fur the $5-\mathrm{km}^{-}$ deep source than it is for the $1-\mathrm{km}$-deep source. In the case of 1 -kin-jeep source, the spectra are dominated by the tectonic component for $F$ values of 1.5 and above. For the 5-km-deep source, the spectra show the effect of the tectonic component at just the lowest frequencies $(00.04 \mathrm{~Hz})$ for $\mathrm{F}$ values af 2. Since Toksöz and Kehrer assuned a surface source, their F values represınt a minimum strength of the tectonic component to produce the effects on this surface waves which they observed. 


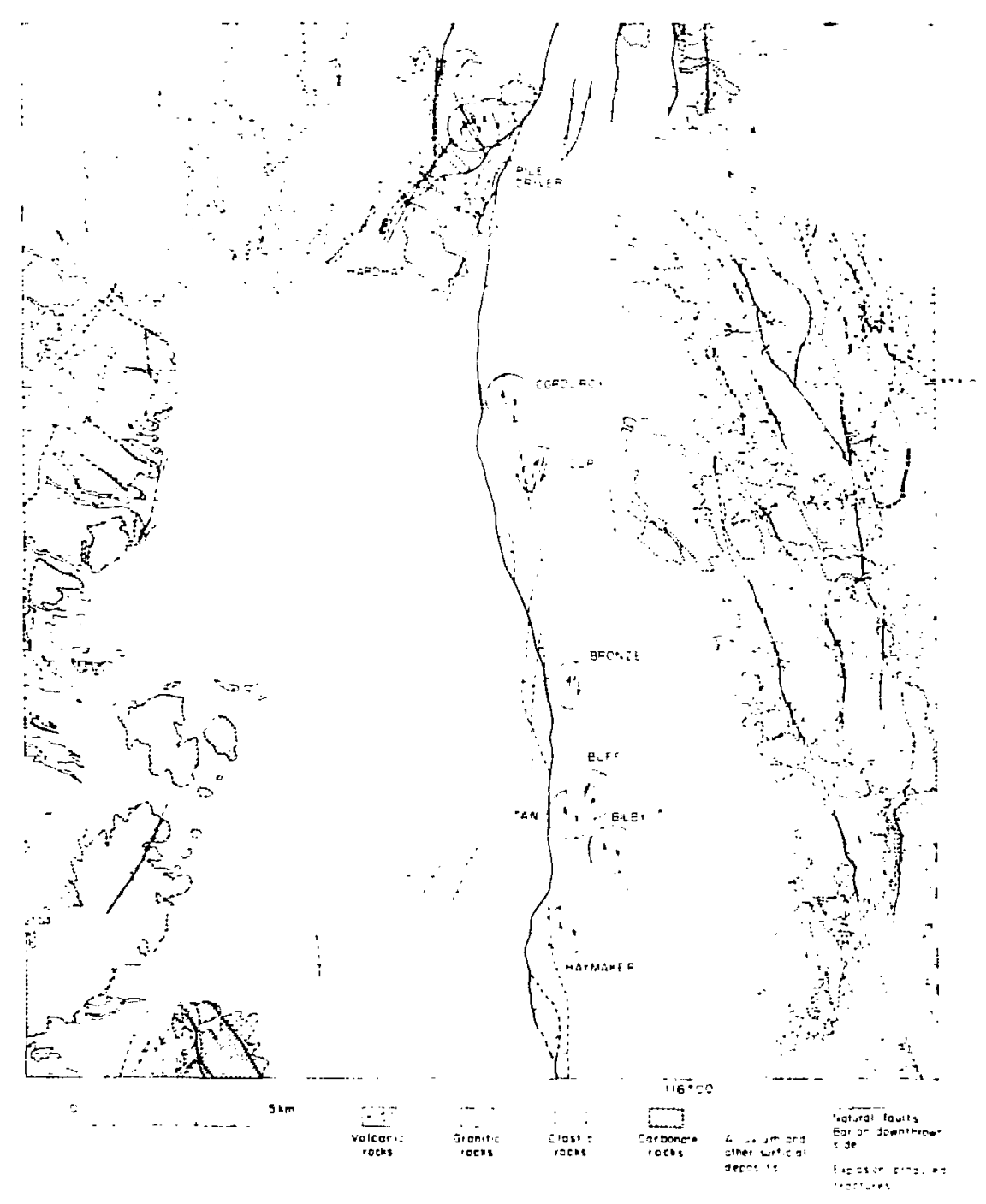

Figure 21. Map of Yucca Valley showing the fault plane orientations of explosion studied by Toksöz and Kehrer. 34 This figure is reproduced from their paper. 

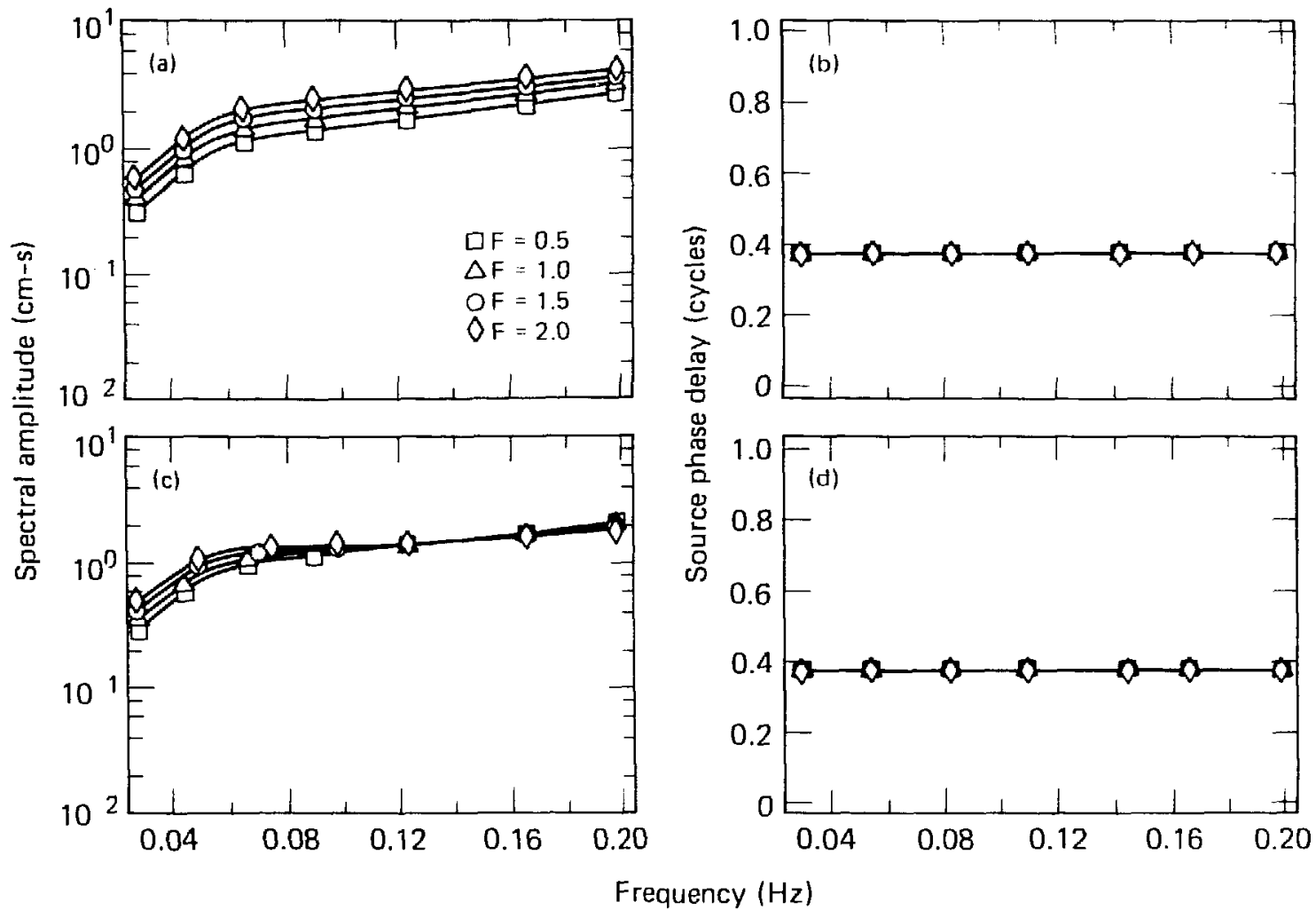

Figure 22. Rayleigh-wave spectra calculated for a multiple-source model proposed by Toksöz and Kehrer ${ }^{34}$ (strike-slip tectonic release superimposed on an explosion). Shown are the (a) amplitude spectra and (b) phase spectra as a function of $F$ value for the case when the tectonic stress release occurs at a depth of $1 \mathrm{~km}$ and the (c) amplitude spectra and (d) phase spectra as a Function of $F$ value for the case when the tectonic stress release occurs at a depth of $5 \mathrm{~km}$. The calculations are for a receiver azimuth corresponding to Mina's. 

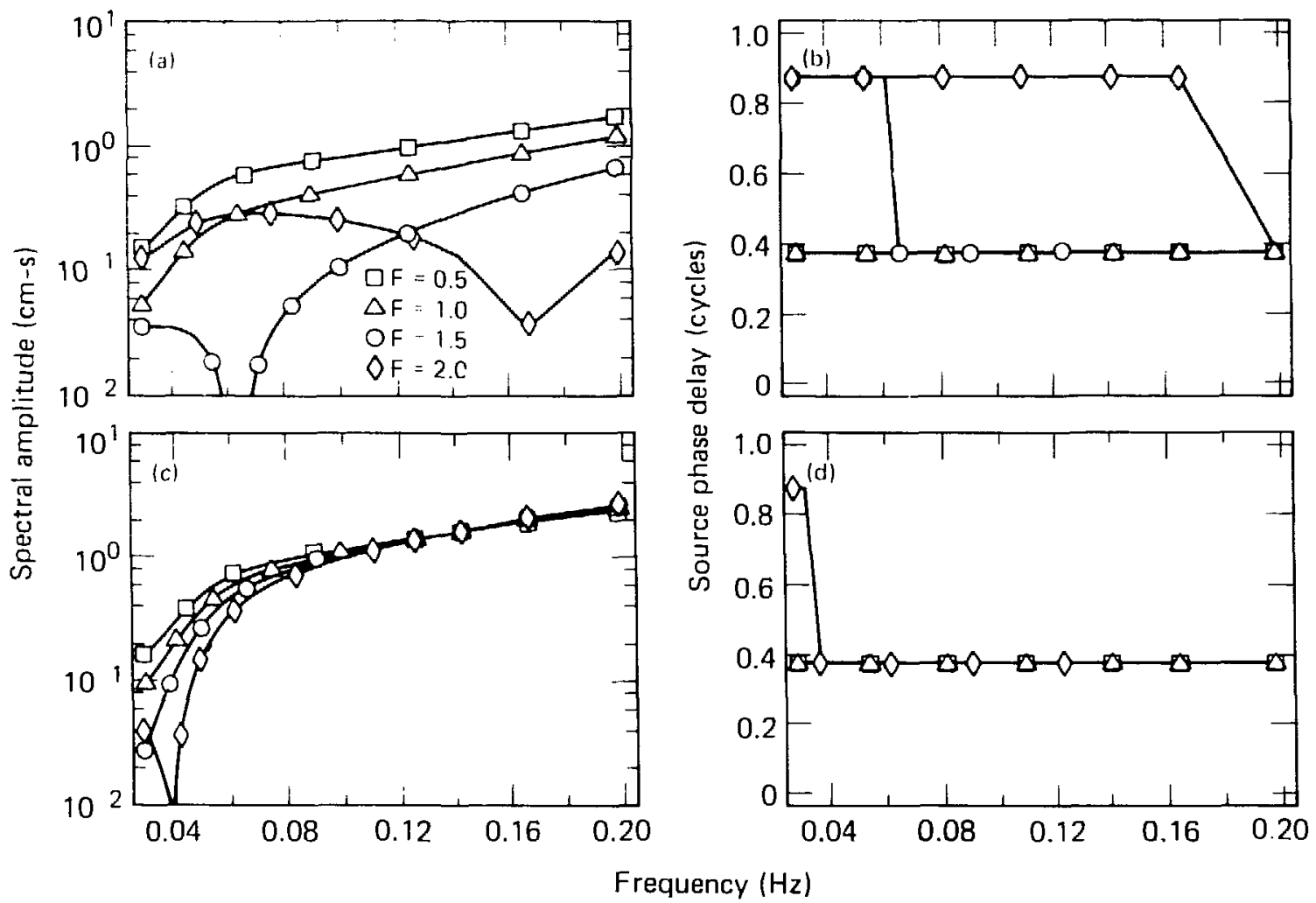

Figure 23. Rayleigh-wave spectra calculated for a multiple-source model proposed by Toksöz and Kehrer ${ }^{34}$ (strike-slip tectonic release superimposed on an explosion). Shown are the (a) amplitude spectra and (b) phase spectra as a function of $F$ value for the case when the tectonic stress release occurs at a depth of $1 \mathrm{~km}$ and the (c) amplitude spectra and (d) phase spectra as a function of $F$ value for the case when the tectonic stress release occurs at a depth of $5 \mathrm{~km}$. The calculations are for a receiver azimuth corresponding to Elko's. 
In applying the Toksöz and Kehrer model to our surface wa'e data, we followed the same trial and error method that was used to estimate fault striks direction and $F$ value for the thrust model. Furthermore, we are able to establish the direction of slip movement on the fault from the Love-wave source-phase data. Indeed, the Love-wave source phase spectra do yield slip directions consistent with the tectonic stress field for three out of the five explosions, i.e., Hearts, Offshore, and Burzet. The source parameters obtained from the data fit are given in Table 6. Examples comparing theoretical spectra to the observed Rayleigh- and Love-wave spectra are shown in Figs. 24 through 32.

DISCUSSION OF MODELING RESULTS

The trial-and-error search for an F-value gave a clear minimum in the RMS residuals for both source models, strike-slip and dip-slip thrust, in the limited range $0 \leq F \leq 1.0$. There were indications that minima existed for F-values greater than 1.0. However, these minima may be discarded because source models with large F-values predict, in some or all azimuths (see Fig. 22 and works of Harkrider, ${ }^{38}$ Massé, $^{3}$ and Patton ${ }^{33}$ ) Rayleigh-wave phase reversals which are not supported by the observations in Fig. 13.

Before discussing the RMS residuals of the Rayleigh-wave fit, we should mention that the Love-wave amplitude and phase observations may be fit equally well by either the strike-slip or the dip-slip thrust model. The only adjistments needed to equalize the fits are to rotate the fault by $45^{\circ}$ and double the seismic moment for the dip-slip event, as many are seen in the third and fourth columns in Table 6.

The surprising result in Table 6 is that the Rayleigh-wave observations can also be fit equally well, or nearly so, for either model when the F-value is less than 1.0. This may be seen by comparing the RMS values of the strikeslip and dip-slip models in Table 6 for each event. Offshore explosion shows the largest contrast $(0.10$ vs 0.16$)$. Yet, in light of the closeness of the calculated spectra shown in Fig. 27 and the uncertainties in the observed spectra, this contrast is not sufficient to discriminate. Aki and $\operatorname{Tsai}^{26}$ demonstrated that discrimination between dip-slip normal Eaulting and strikeslip models was not possible from observations of long-period Rayleigh- and Love-wave amplitudes. Here, we have a similar situation despite observations of both spectral amplitude and phase at higher frequencies. 


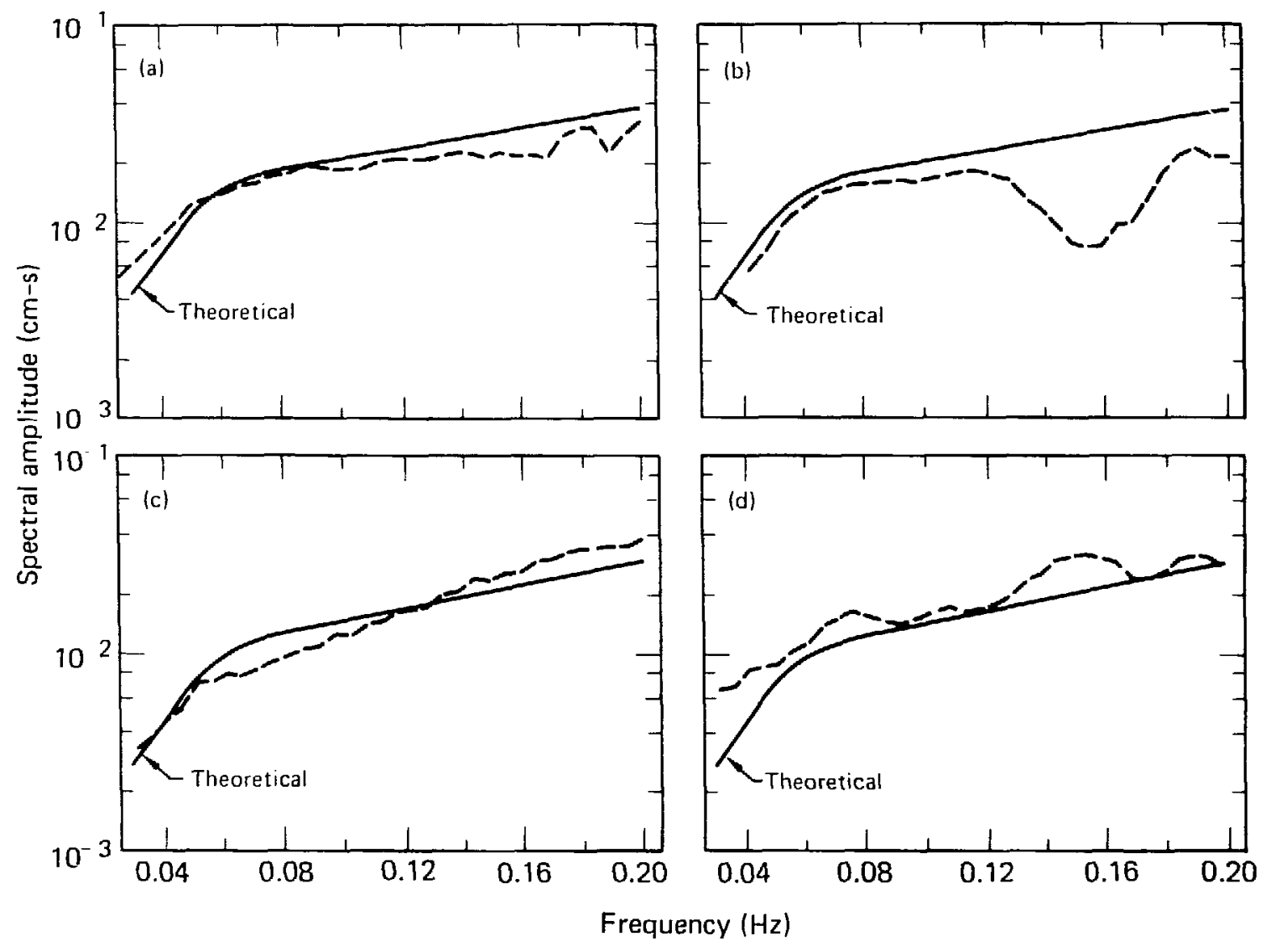

Figure 24. Observed Rayleigh-wave amp'litude spectra for the Hearts' explosion and theoretical spectra computed for the strike-slip model in Table 6 from (a) Mina, (b) Kanab, (c) Landers, and (d) Elko. 


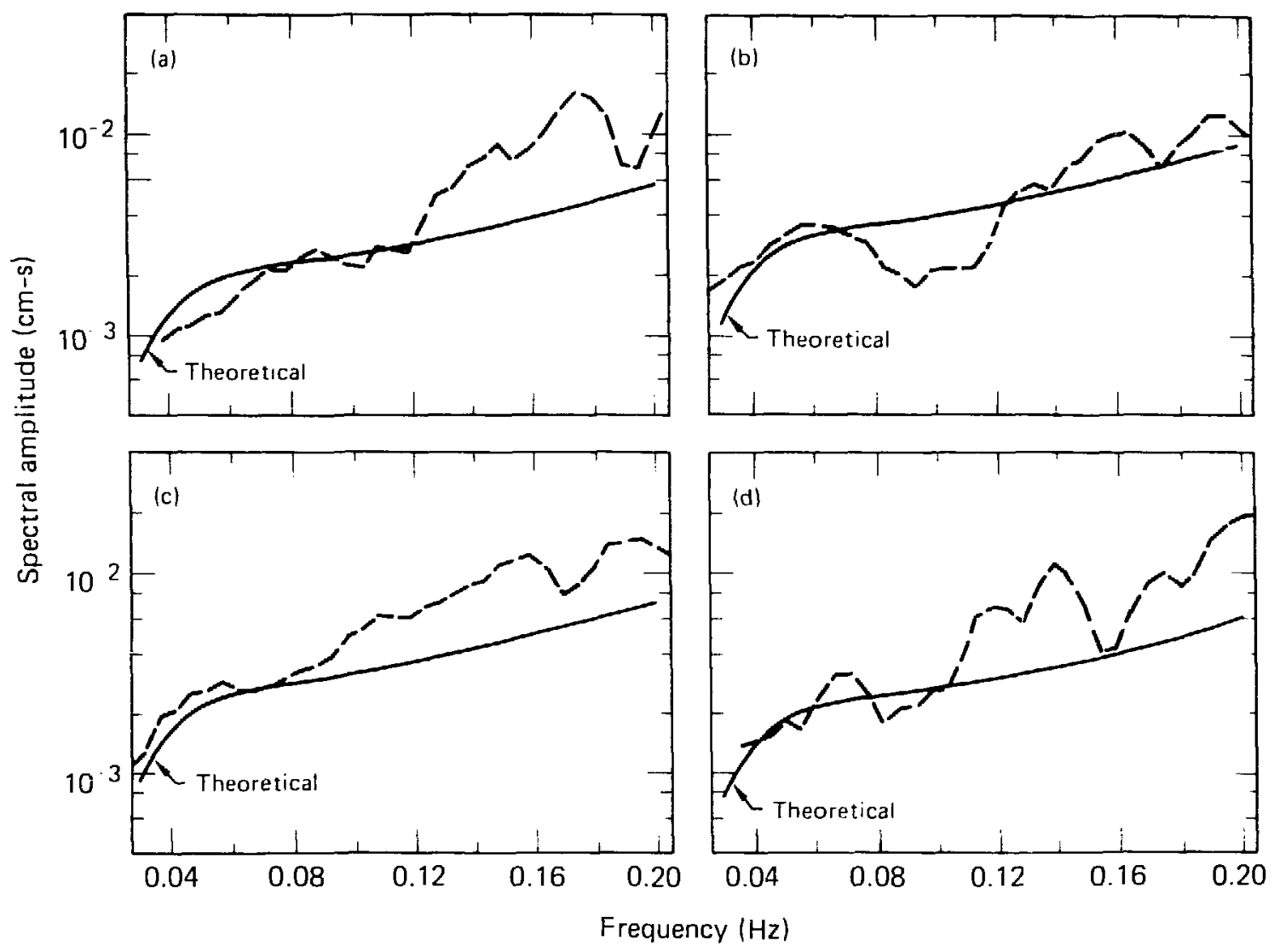

Figure 25. Observed Love-wave amplitude spectra for the Hearts' explosion and theoretical spectra computed for the strike-slip model in Table 6 froin (a) Mina, (b) Kanab, (c) Landers, and (d) Elko.
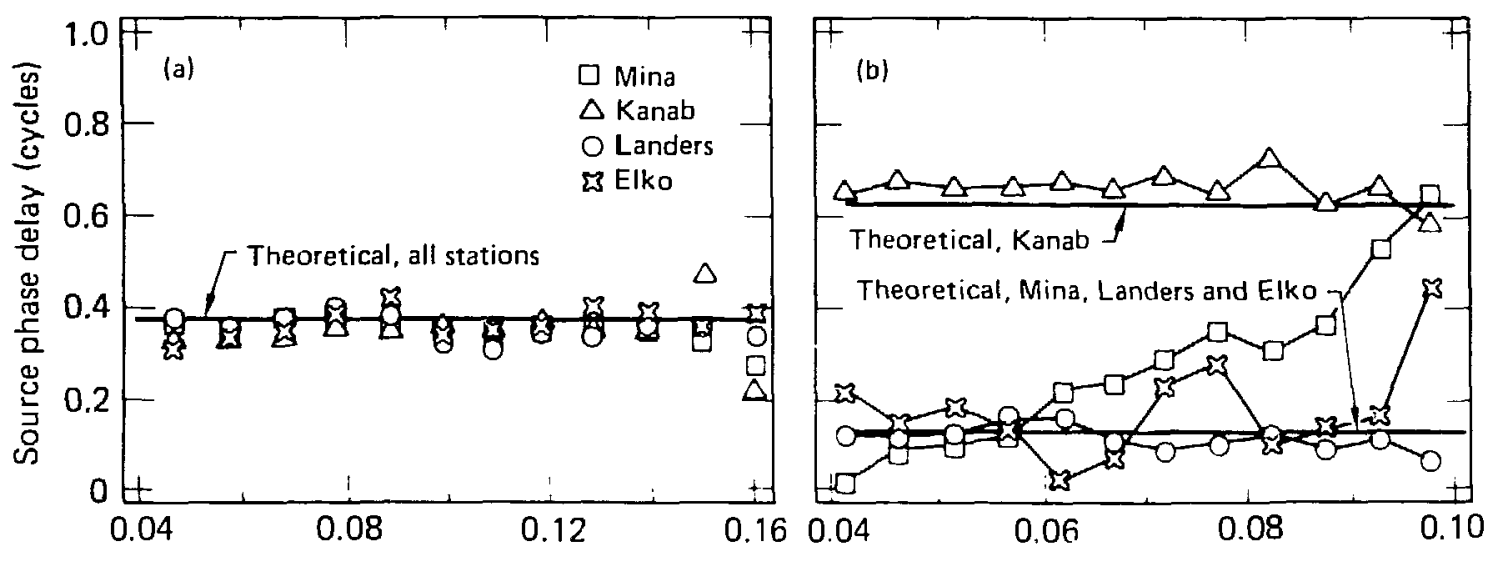

Frequency $(\mathrm{Hz})$

Figure 26. Observed (a) Rayleigh- and (b) Love-wave source phase spectra for the Hearts' explosion and theoretical spectra computed for the strike-slip model in Table 6 . 

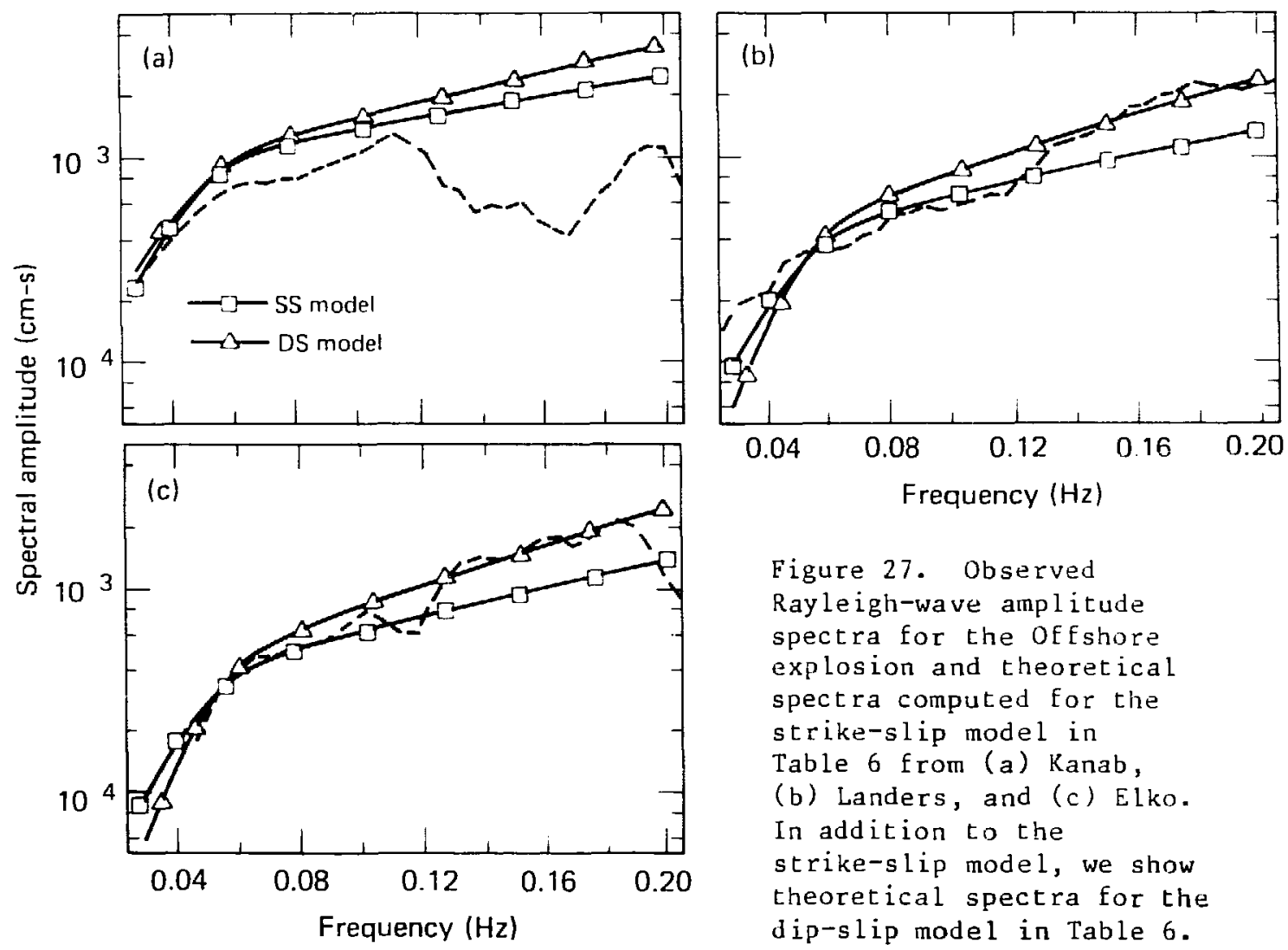

Figure 27. Observed Rayleigh-wave amplitude spectra for the offshore explosion and theoretical spectra computed for the strike-slip model in Table 6 from (a) Kanab, (b) Landers, and (c) Elko. In addition to the strike-slip model, we show theoretical spectra for the dip-slip model in Table 6. 

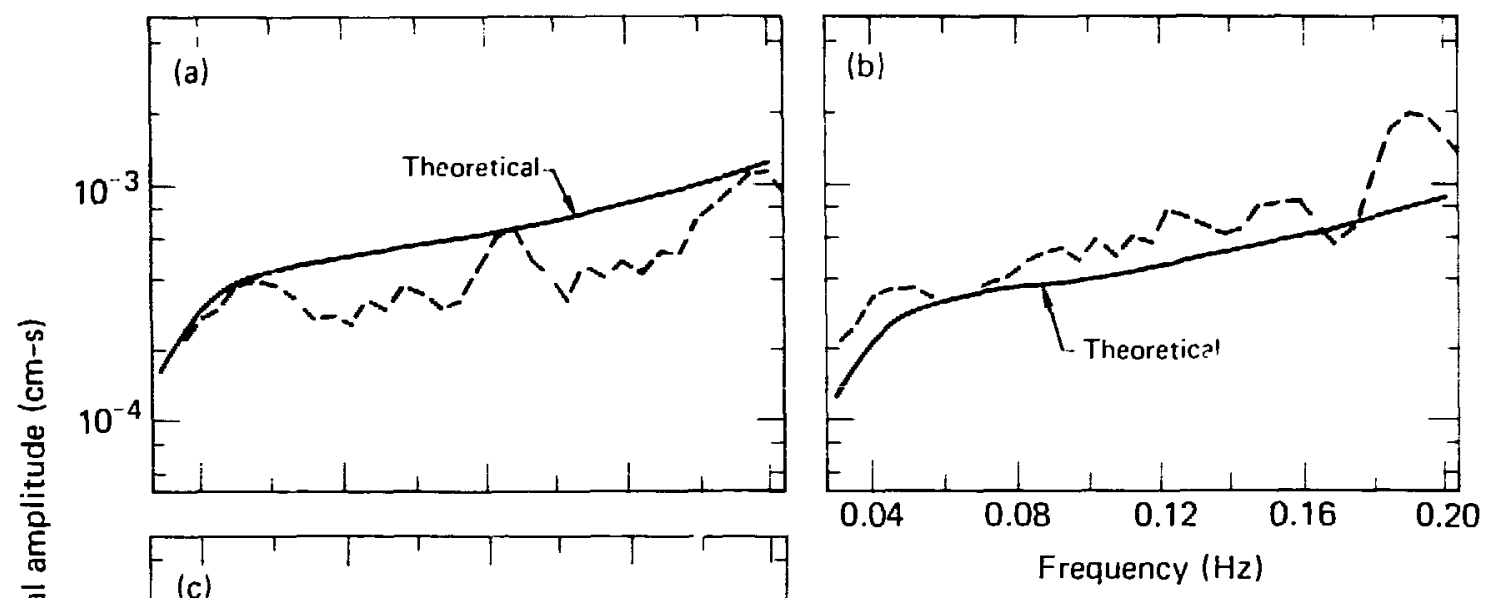

Figure 28. Observed Love-wave amplitude spectra for the offshore explosion and theoretical spectra computed for the strike-slip model in Table 6 from (a) Kanab, (b) Landers, and (c) Elko.
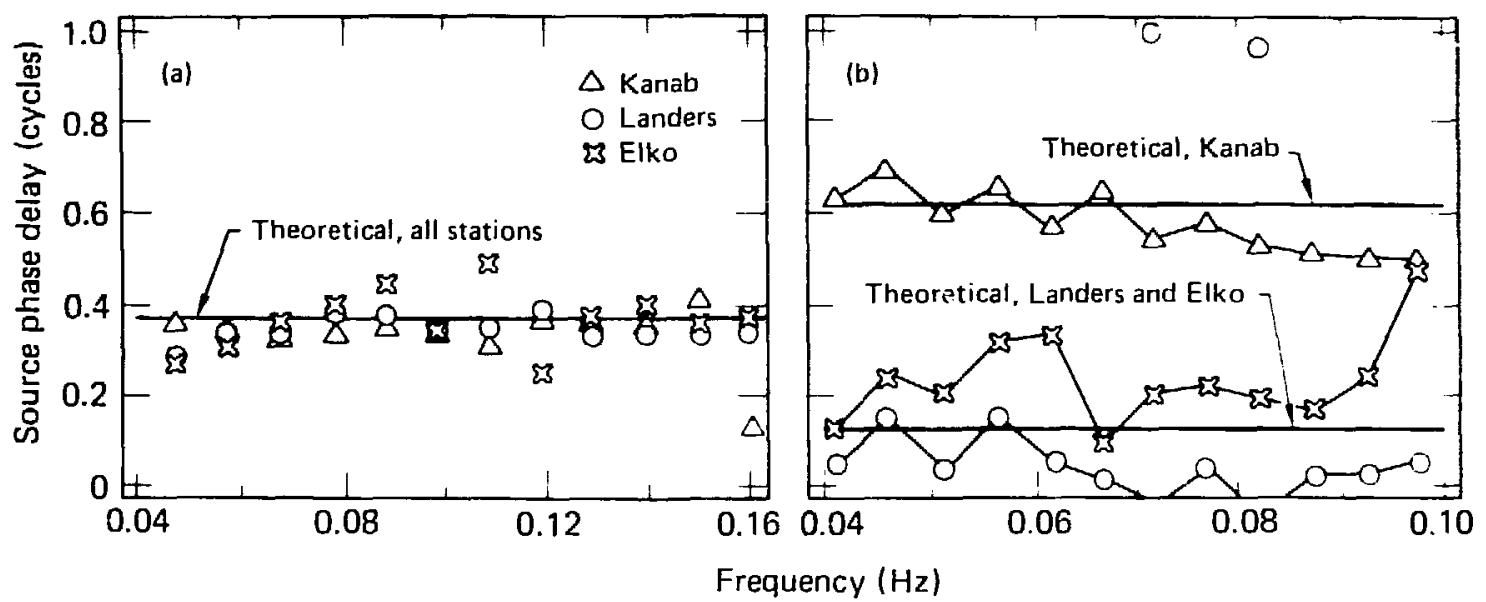

Figure 29. Observed (a) Rayleigh- and (b) Love-wave source phase spectra For the offshore explosion and theoretical spectra computed for the strike-slip model in Table 6 . 


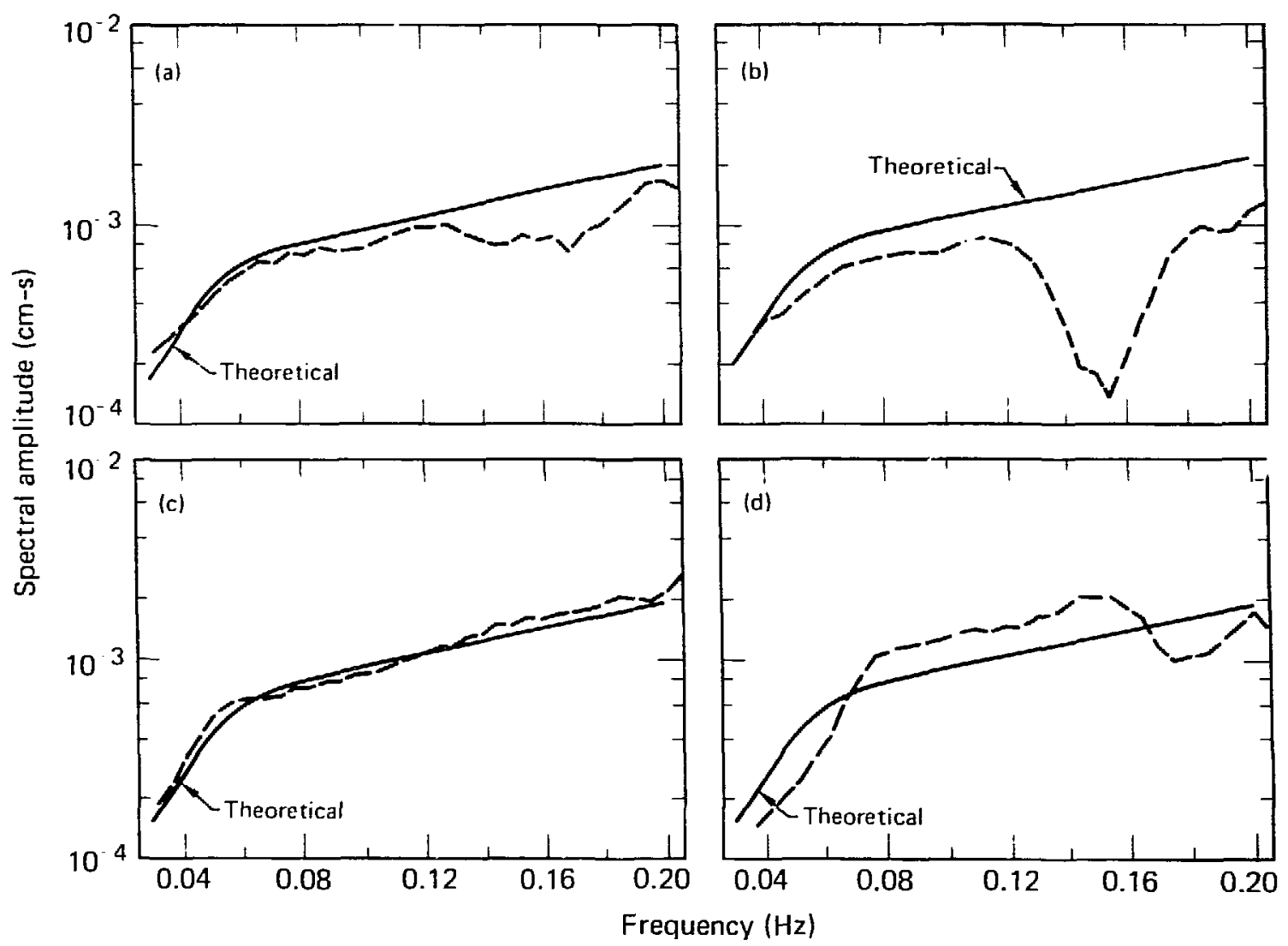

Figure 30. Observed Rayleigh-wave amplitude spectra for the Nessel explosion and theoretical spectra computed for the strike-slip model in Table 6 from (a) Mina, (b) Kanab, (c) Landers, and (d) Elko. 


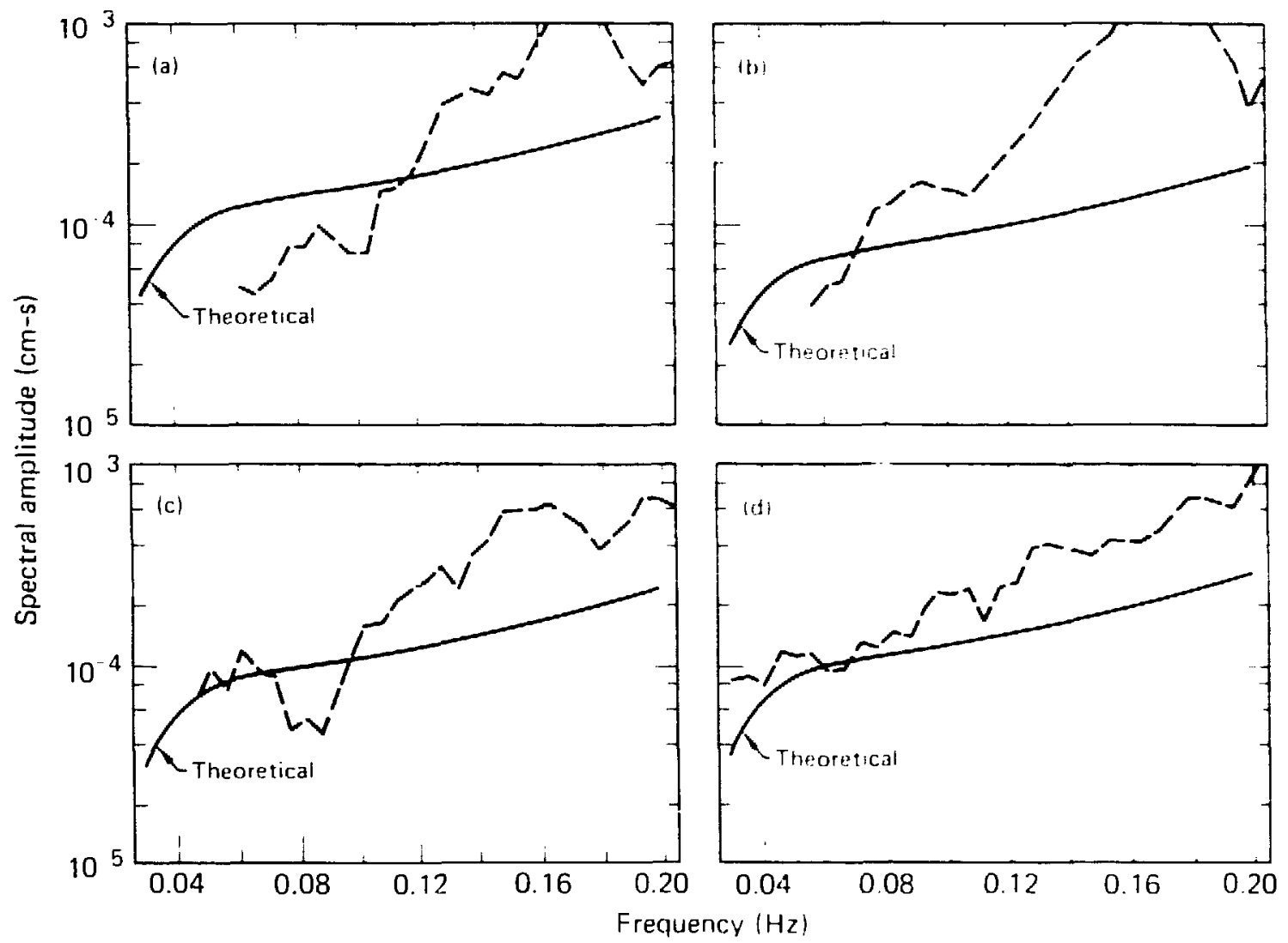

Figure 31. Observed Love-wave amplicude spectra for the thessel txplosion ial theoretical spectra computed for the strike-slip nodel in lasto ir on (a) Mina, (b) Kanab, (c) Landers, and (d) El'oo.
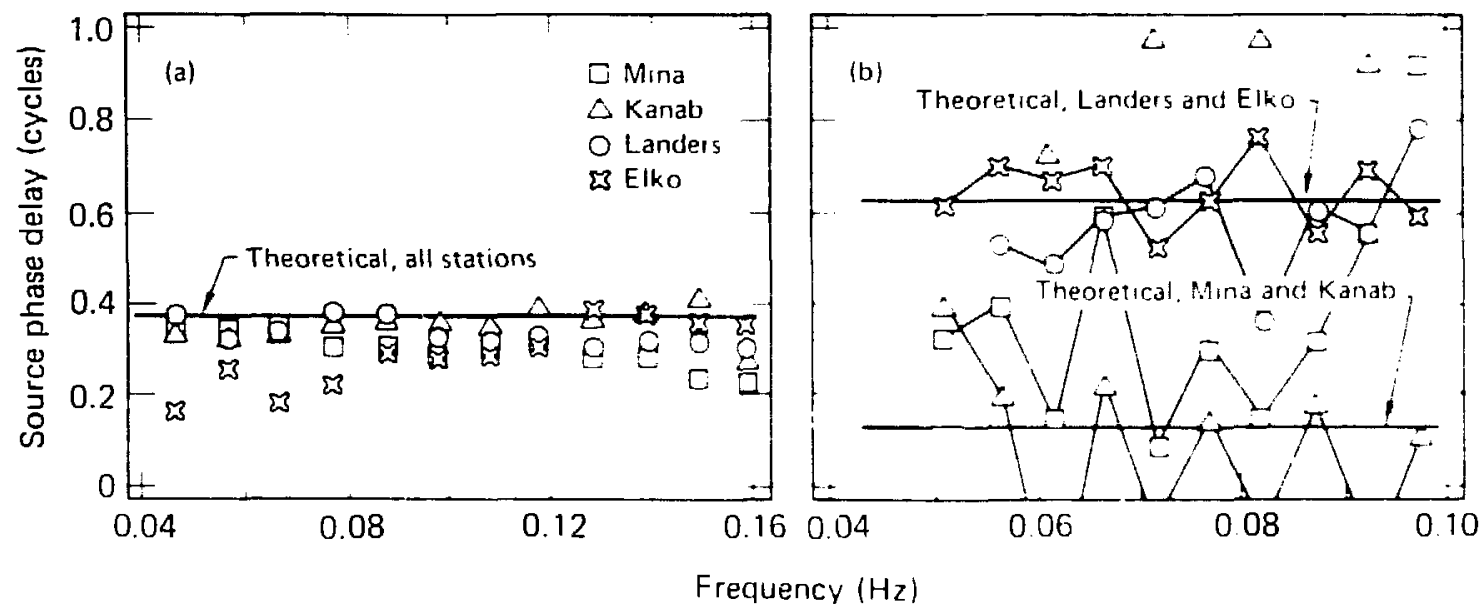

Figure 32. Observed (a) Rayleigh- and (b) Love-wiyg sourze paise spectra iur the Nessel explosion and theoretical spectra computel for the strike-ilip model in Table 6 . 
For explosions heares, Ofisture, and Burzer (possibly Pyramid, although the observations are much pourer) it is tempting to favor the strike-slip model over dip-slip thrust decduse of the results of fitting the Love waves. The estimate of falslt azinuth, $N 20^{\circ} \mathrm{W}$, is consistent with the geologic mapping of the Yuca Faule and nearby splinter faulcs (see Fiz. l). The sense of motion on the fault in the model is right-lateral, which implies a No $5^{\circ} \mathrm{W}$ direction of the leasc-iompressive stress axis. This direction is in goud agreement with the tensional axis of the Massachusetts Mountain Earthquake determined iroin P-wave waves (see Fischer et al. ${ }^{39}$ ) and surface waves (sae pateng.

Althougt the surface-wave observations alone are insufficient to discriminace between strike-slip and dip-slip, tiney do indicate that the secondary source of the Nessel explosion is different from those of the other explosions. Thic is supported by the $40^{\circ}$ rotation in the fault strike rerilired to fit the Love-wave source phase (see Fig. 32). A strike-slip event For chis explosion does not seem to fit well into the picture of an explosion releasing tectonic strain like the other explosions do.

If the secondary source of the Nessel explosion is dip-slip thrust, tine cause is not likely to be tectonic strain release. The least-compressive stress axis is orianted vertically and the maximum compressive stress, inferred iron the faule strike, is $\mathbf{N} 15^{\circ} \mathrm{W}$. We should noce that the strength of Nessel's secondary source is the weakest of all explosions; a seismic moment of $1.9 \times 10^{2 l}$ Jyn-cin corresponds to an earthquake with $M_{L}$ of 3.6 according to the moment-magnitude relationship of Wyss and Bruna. 40

If many $\bar{i}$ aults slipped, as Massé cheorizes in the thrust model, the moment of Nessel's secondary source may be regarded as the sum of moments on all the faults that slipped during the explosion. For simplicity, we assume that there are just two thrust faults extending from the detonation point to the free surface. The moment on each fault is $1.0 \times 10^{21}$ dyn-cm and tire faule length is about $0.5 j \mathrm{~km}(\sqrt{2}$. Nessel's depth ó burial). The average slip, u, on a tault ian be calcalated by Aki's tl formila

$1=4.1$

N Mra $H$ is the $r i g i d i t y$ and $A$ is the fault area. The rigidity of alluvium $i=$ zout $3 \times 10^{10}$ dỵn/cin'. Taking the width of eacin chrust fault to be 
$1 \mathrm{~km}$, we calculate an average displacement of $5 \mathrm{~cm}$. If many more faults are involved, there is not likely to be a clear expression of vertical chrust faulting on the surface near the shot point.

In Fig. 33, we plot body-wave magnitude vs the logaritin of seismic moment for the explosions in this study and for other explosions fired in Yucca Tuff summarized in the study of Aki et al. 27 The scatter of the data on this plot also does not allow the discrimination between dip-slip and strike-slip models. Regardless of which explosion moment we do use, it is evident that the slope of the data on this plot is greater than unity in the range $4.5<\mathrm{m}_{b}<6.0$. In fact, the slope of the data is close to 1.3 . This result appears to be consistent with the empirical $m_{b}: M_{s}$ relationship for NTS explosions reported by Marshall et al. ${ }^{42}$ (the relationship is $\mathrm{M}_{\mathrm{s}}=1.43 \mathrm{mb}_{\mathrm{b}}-3.8$ ) provided that $M_{s}$ is proportional to Log $M_{e}$ for $M_{s}<4.5$.

The proportionality between $M_{s}$ and $\log M_{e}$ for explosions with $M_{S}<4.5$ is predicted from the source spectrum scaling models of both Aki et $a_{1} .^{27}$ and Peppin. 31 The results in the section on Modeling the Explosion Source which concern the low-frequency RDP would imply that the explosion source spectrum is flat or nearly so below $0.2 \mathrm{~Hz}$. This is also predicted from Peppin's model and from the model of Aki et al. 27 for overshoot ratios less chan 2:1. Furthermore, the observations in Fig. 33 suggests that the far-field source spectrum for explosions in tuff with $m_{b}>5$ has a corner (with or without overshoot) at frequencies below $1 \mathrm{~Hz}$. The model of Aki et al. is consistent with these observations. Regarding corner frequencies, Peppin's model appears to be applicable only to near-regional observations which were the basis for this model, since the source spectrum (see Fig. 10 of Ref. 31) does not have a corner frequency less than $1 \mathrm{~Hz}$ even for megaton explosions $\left(m_{b}>6\right)$.

Before concluding our discussion, we should make several comnents about the match of theoretical Love-wave spectra to the observed in Figs. 25, 20, $28,29,31$, and 32. Both the amplitude and the phase spectra are modelej satisfactorily at low frequencies between 0.06 and $0.1 \mathrm{~Hz}$. However, the theoretical spectra do not account for the high spectral amplitudes observed at frequencies above $0.1 \mathrm{~Hz}$.

The shape of the tineoretical ainplitude spectra is atiected by the inediuin model, source parameters (deptn and orientation of the double-couple), and the $t$ ime function of the source. The average velocity model in Table $i$ is probably a good estimate of the best plane-layered model representing the medium between 


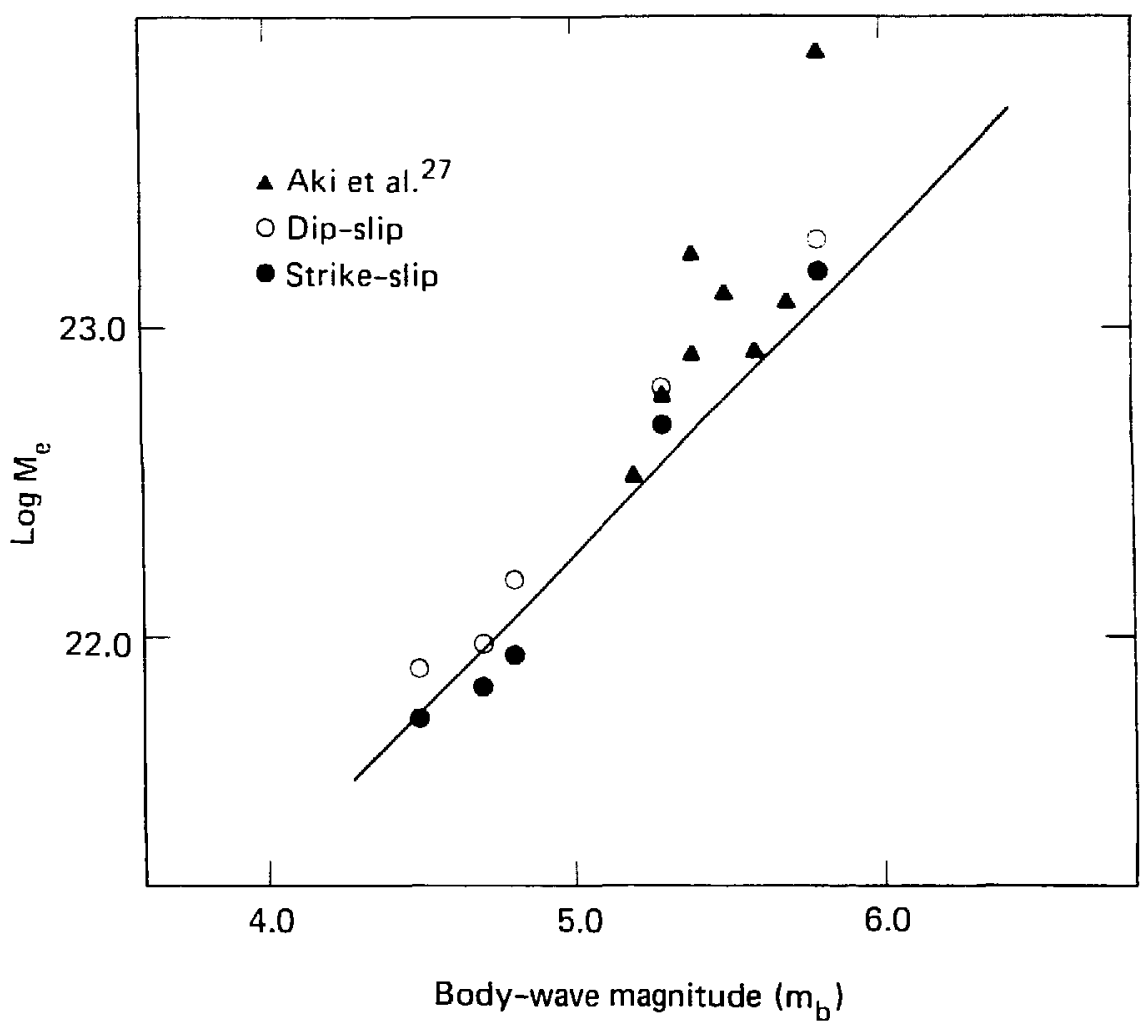

Figure 33. A plot of $\mathbf{m}_{b}$ (given in Table 1 ) vs logarithm of seismic moment of axplosions studied in this paper and in the paper by Aki et al.27 A line with unity slope is shown on this plot for reference. 
NTS and the LLNL stations. Further refinements of the plane-layered model are not likely to enhance the excitation of high frequencies substantially. Source parameters, such as focal depth, affect the Love wave excitation; however, the amplitudes at high frequencies are reduced rather than enhanced as the focus moves deeper (see Figs. 2 and 3 of Tsai and $\mathrm{Aki}^{16}$ ) For shallow sources, the orientation of the double-couple does not change the shape of the amplitude spectrum greatly.

More high Erequencies could be introduced into the source model through the source time function of the secondary source. This is completely analogous to the effect of the RDP on the Rayleigh-wave spectra of the explosion. An impulse time function would increase the slope of the excitation spectrum by w over that of a step function. However, the improvement that would result in the fit to the Love-wave amplitude spectra would be offset by a loss of $f$ it

to the Love-wave phase spectra.

We speculate that there ina be more than one source of Love-waves in the Erequency range 0.06 to $0.16 \mathrm{~Hz}$. The observations at low Erequencies $(<0.1 \mathrm{~Hz})$ are modeled well by the double-couple source with step function time dependence, the source representation proven to be appropriate for earthquake strain release. However, this suurce model does not account for the enhanced amplitudes at high frequencies. The implication is that another source dominates at high frequencies $(>0.1 \mathrm{~Hz})$. Furthermore, we speculate that this source must be very inefficient at generating low frequency seismic waves.

\section{CONCLUSIONS}

In this paper we have reported on surface-wave studies of five underground nuclear explosions detonated in Yucca Valley. We draw the following conclusions:

\footnotetext{
1. The amplitudes of explosion-generated Rayleigh waves in the frequency range 0.03 to $0.16 \mathrm{~Hz}$ are modeled well by an RDP with little or no overshoot. This result holds for explosions in alluvium and cuff, above and below the water table.
} 
2. For explosions in this study, spall closure as pruposed by viecelli ${ }^{22}$ does not appear to be a significant source of Rayleigh waves that reach the Ear-field.

3. There are secondary sources of seismic waves for all explosions as indicated by the presence of Love waves and non-istropic Rayleigh-wave a:nplitude and phase spectra.

4. The observations of Love- and Rayleigh-wave amplitudes and source phases alone are not sufficient to discriminate between the strike-slip model and dip-slip thrust model at the Low F-values ( 0.2 to 0.3$)$ obtained for these explosions. In the case of the strike-slip model, the principal axes inferred from the fault slip and strike are in good agreenent with the regional tectonic stress field for all but one explosion, Nessel. The results from the Nessel explosion suggest a mechanism other than tectonic strain release. Unfortunately, there is little hope to confirm that Nessel's source is different from the other explosions from field observations of surface faulting.

FUTURE WORK

This paper has reported on progress in our research carried out over the past year or so. There is a need to continue work on all aspects of this research. We take the opportunity here to mention a few areas of future work.

\section{Augmented Datasets}

We plan to augment the present dataset with three-component digital data Erom Sandia Laboratory's seismic station network surrounding NTS. This will allow a closer examination of the azimuthal variation in seismic wave generation which, in turn, will result in better constraints on the deviatoric part (non-explosive part) of the source description.

RDP at Higher Frequencies

We plan to continue work on the RDP by studying the $\mathrm{L}_{\mathrm{g}}$ phase. This relatively "broad-band" phase will provide information at higher frequencies 
than the fundamental-mode surface waves do and, yet, should overlap sufficiently to tie into our low-frequency description of the RDP.

\section{Love-wave Generation}

We plan to pursue the finding of enriched Love-wave spectral amplitudes at high frequencies by including additional observations of explosions and earthquakes in the western U.S. If the enriched amplitudes are caused by a source effect, we may see differences between Love waves from earthquakes and explosions.

\section{Improved Inversion Techriques}

We plan to improve on the inversion techniques for source parameters by implementing automated inversion schemes and methods to model larger datasets simultaneously.

\section{ACKNOWLEDGMENTS}

Many individuals of the seismic groups at LLNL aided and are continuing to aid either directly or indirectly with the progress of this work. We wish to thank in particular: N. Burr for assembling the dataset of Lawrence Livermore National Laboratory seismograms, K. Nakanishi and S. Taylor for beneficial discussions, D. Springer for encouragement throughout this project, and J. Miracle for typing numerous revisions of the manuscript. J. Hannon, K. Nakanishi, P. Moulthrop, and S. Taylor read the manuscript and offered valuable criticisms. 
APPENDIX A.

PROCEDURES FOR ANALYSIS TECHNIQUES

The purpose of this appendix is to present the detailed procedures for each of the following analysis techniques: (1) low-pass filtering, (2) narrow-band filtering, and (3) Fourier spectral analysis. Explicit mathematic formulas referenced in these procedures are given at the end of the appendix.

PROCEDURE FOR THE LOW-PASS FILTERING

STEP 1. Time windows were selected to include vertical $\mathrm{Lg}$ and Rayleigh waves and transverse Lg and Love waves. Table 2 gives the time windows for each component and station.

STEP 2. The waveform data were windowed with a boxcar function with the number of points equal to the closest power of two.

STEP 3. The windowed data were fast Fourier transformed. Amplitude and phase spectra were computed (see Definition of Amplitude and Phase Delay Spectra).

STEP 4. Low-pass filter (see Cosine Squared Taper for Low-Pass Filtering) was applied to the amplitude spectrum.

STEP 5. The filtered waveform was obtained by performing an inverse FFT.

PROCEDURE FOR NARROW-BAND FILTERING

STEP 1. Time windows were selected to include all arrivals having velocities slower than about $3.9 \mathrm{~km} / \mathrm{s}$.

STEP 2. The waveform data were windowed with a $20 \%$ cosine taper function (see Cosine Window for Shaping $f(t)$ before Transforming) and zero filled to a power of two points. 
STEP 3. The windowed data were fast Fourier transformed. Amplitude and phase spectra were computed.

STEP 4. The narrow-band Filter (see Narrow-Band Filter) was applied to the amplitude spectrum.

STEP 5. The filtered waveform was obtained by computing the inverse FFT.

PROCEDURE FOR SPECTRAL ANALYSIS OF SIGNALS AND NOISE SAMPLES

STEP 1. Time windows were selected to include the surface wave arrivals. These windows are given in Table 3. Noise windows, the same length as the signal windows, were selected. The windows were taken before the onset of $P$ waves in the time period -125 to 25 s relative to the detonation time $(t=0.0)$.

STEP 2. The waveform data were windowed with a $10 \%$ cosine taper function (see Cosine Window for Shaping $f(t)$ before Transforming) and zero filled to the closest power of two points.

STEP 3. The windowed data were fast Fourier transformed. Amplitude and phase spectra were computed (see Definition of Amplitude and Phase Delay Spectra).

STEP 4. The transfer function of the LLNL seismological system was removed to obtain amplitute and phase spectra of ground displacement. We used the analytic form of the transfer function (see Nakanisini ${ }^{43}$ ).

DEFINITION OF AMPLITUDE AND PHASE DELAY SPECTRA

Let $f(t)$ be a seismogram record of interest in the time frame $t \leq t \leq t_{2}$. We compute the fourier transform, $F(\omega)$, of the record as follows:

$F\left(\omega_{k}\right)=A\left(\omega_{k}\right) e^{-i \phi(\omega)_{k}}=\int_{t_{1}}^{t} f(t) e^{-i \omega_{k} t} d t$, 
where $\omega_{k}$ is a particular frequency. If $\operatorname{Re}\left(F_{k}\right)$ refers to the real part of $F$ at the frequency $\omega_{k}$ and $\operatorname{Im}\left(F_{k}\right)$ refers to the imaginary part, then

$A\left(\omega_{k}\right)=\sqrt{\operatorname{Re}\left(F_{k}\right)^{2}+\operatorname{Inn}\left(F_{k}\right)^{2}}$

and

$\phi\left(\omega_{k}\right)=\operatorname{TAN}^{-1}\left[\frac{-\operatorname{Lm}\left(F_{k}\right)}{\operatorname{Re}\left(F_{k}\right)}\right]$,

where $A\left(\omega_{k}\right)$ is the amplitude spectrum and $\phi\left(\omega_{k}\right)$ is the phase delay spectrum.

COSINE-SQUARED TAPER FOR LOW-PASS FILTERING

Let $\Delta \omega$ be a constant frequency increment and $\omega_{c}$ be a corner frequency. Let $N$ (an odd integer) be the number of frequency points in the taper. The frequency limits of the taper are

$\omega_{L}=\omega_{C}-\Delta \omega \cdot \frac{i j-1}{2}$

and

$\omega_{H}=\omega_{c}+\Delta \omega \cdot \frac{N-1}{2}$.

Then, the functional form of the taper operator, $T(\omega)$, is as follows:

$T(\omega)= \begin{cases}1 & \omega<\omega_{L} \\ \cos ^{2} \frac{\pi\left(\omega-\omega_{L}\right)}{2\left(\omega_{H}-\omega_{L}\right)} & \omega_{L} \leq \omega \leq \omega_{H} \\ 0 & \omega>\omega_{H} .\end{cases}$ 
Let $\Delta t$ be a constant time increment and $N$ be the total number of points in the interval $t_{1} \leq t \leq t_{2}$. If $\mathrm{P}$ refers to the percent of the interval with cosine taper, then $\mathrm{N} \cdot \mathrm{P} / 100$ is the number of points affected by the taper. Let $M$ be the total number of points affected by the taper. Of this number, half are affected at the beginning and half are affected at the end. We define new times, $t_{3}$ and $t_{4}$, as

$t_{3}=t_{1}+\Delta t \cdot \frac{M}{2}$

and

$t_{4}=t_{2}-\Delta t \cdot \frac{M}{2}$

Then, the functional form of the window operator, $W(t)$, is as follows:

$$
W(t)= \begin{cases}\frac{1}{2}\left[1-\cos \frac{\pi\left(t-t_{1}\right)}{\left(t_{3}-t_{1}\right)}\right] & t_{1} \leq t \leq t_{3} \\ 1 & t_{3} \leq t \leq t_{4} \\ \frac{1}{2}\left[1+\cos \frac{\pi\left(t-t_{4}\right)}{\left(t_{2}-t_{4}\right)}\right] & t_{4} \leq t \leq t_{2} .\end{cases}
$$

NARROW-BAND FILTER

The design of a narrow-band filter used in this study is the same as Dziewonski et al. ${ }^{6}$ Let $\omega_{c}$ be the center frequency. The functional form of the narrow-band filter is as follows:

$$
H(\omega)= \begin{cases}0 & w<(1-\text { BAND }) \cdot \omega_{c} \\ e^{-a\left(\omega-\omega_{c}\right)^{2} / \omega_{c}} & (1-\text { BAND }) \cdot \omega_{c} \leq \omega \leq(1+B A N D) \cdot \omega_{c} \\ 0 & w>(1+B A N D) \cdot \omega_{c},\end{cases}
$$


where BAND is the bandwidth of the filter and $\alpha$ is a parameter controlling the amplitude at the cut-off frequencies. Specifically, we introduce a parameter, $B$, such that

$B=-\ln \left[H\left(\omega_{L}\right)\right]=-\ln \left[H\left(\omega_{H}\right)\right]$,

where

$$
\begin{aligned}
& \omega_{L}=(1-\text { BAND }) \cdot \omega_{c} \\
& \omega_{H}=(1+\text { BAND }) \cdot \omega_{c}
\end{aligned}
$$

and

$$
\alpha=B / \text { BAND }^{2}
$$

Satisfactory results are obtained when BAND equals 0.5 and $B$ equals 3.15 . 
1. G. Müller, "Seismic Moment and Long-Period Radiation of Underground Nuclear Explosions," Bull. Seism. Soc. Am. 63, 847 (1973).

2. D. Springer, "Secondary Sources of Seismic Waves from Underground Nuc lear Explosions," Bull. Seism. Soc. Arn. 64, 581 (1974).

3. R. Massé, "A Seismic Source Model for Underground Nuclear Explosions," Bull. Seism. Soc. Am. 71, 1249 (1981).

4. M. Denny, The Installation of Horizontal Seismometers in the Lawrence Livermore Laboratory Seismic Network and Their Calibration, Lawrence Livermore National Laboratory, Livermore, CA, UCRL-52216 (1977).

5. Surficial Geologic Map of Yucca Flat, Nye, and Lincoln Counties, Nevada, U.S. Geological Survey, Map No. I-550 (1968).

6. A. Dziewonski, S. Block, and M. Landisman, "A Technique for the Analysis of Transient Seismic Signals," Bull. Seism. Soc. Am. 59, 427 (1969).

7. P. Rodgers, $M$. Denny, and $M$. Humme 11 , Transfer Function, Frequency Response, Phase and Group Delays for DS ${ }^{2}$, Lawrence Livermore Nationai Laboratory, Livermore, CA, internal memorandum DET 80-4, (April 3, 1980).

3. H. Patton, Measurements of Rayleigh-Wave Phase Velocicies in Nevada: Implications For Explosion Sources and the Massachusetts Mountain Earthquake, Lawrence Livernore National Laboratory, Livermore, CA, UCRL-86105 ( 1981 ).

9. K. Priestley and J. Brune, "Surface Waves and the Structure of the Great Basin of Nevada and Western Utah," J. Geophys. Res. 83, 2265 (1978).

10. A. E. H. Love, A Treatise on the Mathemacical Theory of Elasticity (Dover Publications, New York, 1944).

11. G. Miiller, "Theoretical Seismograms for Some Types of Point-Sources in Layered Media--Part III, Single Force and Dipole Sources of Arbitrary Orientation," Z. Geophys. 35, 347 (1969).

12. Y. B. Tsai and K. Aki, "Amplitude Spectra of Surface Waves from Snall Earthquakes and Underground Nuclear Explosions," J. Geophys. Res. 76 , 3940 (1971).

13. 4. Saito, "Excitation of Free Oseillations and Surface Waves by a Point Source in a Vertically Heterogeneous Earth," J. Geophys. Res. 72, 3639 $(1957)$. 
14. H. Takeuchi and M. Saito, "Seismic Surface Waves," Methods in Lomputational Physics, B. A. Bolt, Ed. (Academic Press, New York, NY, 1972), pp. 217-295.

15. D. Vun Seggren and R. Blandford, "Source Time Functions and Spectra for Underground Nuclear Explosions," Geophys. J. R. Astr. Soc. 31, 83 (1972).

15. Y. B. Tsai and K. Aki, "Precise Focal Depth Determination from Amplitude Spectra of Surface Waves," J. Geophys. Res. 75, 5729 (1970).

17. E. Carpenter, R. Savill, and J. Wright, "The Dependence of Seismic Signal Amplitudes on the Size of Underground Explosions," Geophys. J. R. Astr. Soc. 6 , $426(1962)$.

13. G. Nerth and R. Herbst, "Comparisons of Amplitudes of Seismic Waves from Nuclear Explosions in Four Mediums," J. Geophys. Res. 68, 1463 (1903).

19. R. Blandford, Experimental Determination of Scaling Laws for Contained and Cratering Explosions, Seismic Data Analysi.s Center, Alexandria, VA, Report No. 76-3 (1976).

20. J. Hudson and A. Douglas, "Research Note on the Amplitudes of Seismic Waves," Geophys. I. R. Astr. Soc. 42, 1039 (1975).

21. C. Prodenl, Crustal Structure of the Western United States, U.S. Geological Survey Professional Paper No. 1034 (1979).

22. J. Viecelli, "Spallation and the Generation of Surface Waves by an Underground Explosion," J. Geophys. Res. 78, 2475 (1973).

23. J. Brune and P. Poneroy, "Surface-Wave Radiation Patterns for Underground Nuclear Explosions and Small Magnitude Earthquakes," J. Geophys. Res. ó8, $5005(1963)$.

24. K. Aki, "A Note on Surface Waves from Hardhat Nuclear Explosion," J. Geophys. Res. 69, 1131 (1964).

25. K. Aki, P. Reasenberg, T. Defazio, and Y. Tsai, "Near-Field and Far-Field Seismic Evidences for Triggering of an Earthquake by the Benham Explosion," Bull. Seism. Soc. Am. 59, 2197 (1969).

26. K. Aki and Y. B. Tsai, "Mechanism of Love-Wave Excitation by Explosive Sources," J. Geophys. Res. 77, 1452 (1972).

27. K. Aki, M. Bouchon, and P. Reasenberg, "Seismic Source Function of an Underground Nuclear Explosion," Bull. Seism. Soc. Am. 74, 131 (1974).

23. J. Eisler, F. Chilton, and F. Sauer, Multiple Subsurface Spalling by Underground Nuclear Explosions," J. Geophys. Res. 71, 3923 (1960).

29. S. Warshaw, Lawrence Livermore National Laboratory, Livermore, CA, private communication (November 1980). 
30. T. Bache, $W$. Rodi, and $B$. Mason, Source Amplizudes di ils Explosions from Rayleigh Waves at Albuquerque and Tucson, science, jysterns, and joftware, La Jolla, CA, Technical Report SSS-R-78-3090 (1978).

31. W. Peppin, "A Near-Regional Explosion Souree Model for Túf," Geophys. J. R. Astr. Soc. 48, 331 (1977).

32. F. Mckeown and D. Dickey, "Fault Displacements and Motion Related co Nucleat Explosions," Bull. Seism. Soc. Am. 59, 2253 (1969).

33. H. Patcon, Surface-dave Generation by Underground Nuclear Explosions Releasing Tectonic Strain, Lawrence Livernore iational Laboratory, Livermore, CA, UCRL-53062 (1930).

34. M. i. Toksioz and H. kehrer, "Tectonic strain Release by underground Nuclear Explosions and Its Effect on Beisnic Discrinination," Geopigs. j. R. Astr. Soc. $31,141(1972)$.

35. F. Press and C. Archambeau, "Release of rectonic itrain by Underground Nuclear Explosions," J. Geopiys. Res. 07, 337 (1962).

36. C. Archambeau and C. Sammis, "Seismic Kaciation Éron Explosions in Prestressed Media and che Measurenent of Tectonic jeress in the ëartn," Rev. Geophys. Space Phys. 8, 473 (1970).

37. M. Zoback and Y. Zoback, "State of stress in the Conterminous United States," J. Geophys. Res. 35, 1113 (1930).

38. D. Harkrider, "Ray and hode Modeling of Seisnic 'vaves ar Rezl,nal Distances," in Identification of jeismic sources, Earthquáe ue Underground Explosion, E. S. Husebye and S. Hykkeltueic, Eds. (D. Raidai Publishing Co., Dortrecht, The Netherlands, 1981).

39. F. Fischer, P. Papanek, and R. Hamilton, The Massachusetts - Hountain Earthquake of 5 August 1971 and Its Aftershocks, Nevada Test Site, U.S. Geological Survey, Report ivo. 474-149 (1972).

40. M. Wyss and J. Brune, "Seismic Moment and Source Dimensions for Earthquakes in the California-Nevada Region," J. Geophys. Res. 73, 4031 (192s).

4l. K. Aki, "Generation and Propagation of $G$ - Javes Éruin idigata Ëarthquake of June 16, 1964," Bul1. Earthquake Res. Inst. 44, 73 (19.9.).

42. 2. Marshall, D. Springer, and H. Kudean, "Lagnituie Corrections iur Attenuation in the Upper Mantle," Geophys. J. R. Astr. Soc. 37 , njo (197y).

43. K. Nakanishi, Computer Code for the Transfer Function or seismiz jystems, Lawrence Livermore National Laboratory, Livermore, CA, UClD-lBuTl (lyty). 